\title{
Catalogue of vibration reduction index formulas for heavy junctions based on numerical simulations
}

\author{
Jordi Poblet-Puig*1 and Catherine Guigou-Carter ${ }^{\dagger 2}$ \\ ${ }^{1}$ Laboratori de Càlcul Numèric, E.T.S. d'Enginyers de Camins, Canals i \\ Ports de Barcelona, Universitat Politècnica de Catalunya \\ ${ }^{2}$ Centre Scientifique et Technique du Bâtiment
}

May 6, 2017

\begin{abstract}
The vibration reduction index $\left(K_{i j}\right)$ is a key parameter in the prediction of flanking transmissions according to the EN-12354 standard. Formulas for the evaluation of $K_{i j}$ in $\mathrm{L}, \mathrm{T}$ and $\mathrm{X}$ junctions that depend on the mass ratio are available in the Annex E. Junctions of straight elements with different thickness or thin elastic layers are also included. However, other junction types that are important for building industry are not considered: H-shaped junctions, L or T junctions not forming a right angle, asymmetrical T-junctions, X-junctions where only one of the parts is different (thickness or material) from the other two/three. In the current research, expressions for these non-covered junctions are provided. They are obtained by means of numerical simulations based on the spectral finite element method. $K_{i j}$ is predicted for a large population of junctions, considering usual thicknesses and heavy material combinations (no lightweight frame systems have been considered). Statistical analysis is carried out to obtain relatively simple formulae that could be used in acoustic design projects without the need for time-consuming computations with finite element software.
\end{abstract}

Keywords: junction, $K_{i j}$, EN-12354, SFEM

\footnotetext{
*correspondence: UPC, Campus Nord B1, Jordi Girona 1, E-08034 Barcelona, Spain, e-mail: jordi.poblet@upc.edu

${ }^{\dagger}$ correspondence:24 rue Joseph Fourier, 38400 Saint Martin d'Hères, France, e-mail: catherine.guigou@cstb.fr
} 


\section{Introduction}

An important part of the sound transmitted between rooms in a building passes through indirect paths. There are often multiple paths which cause flanking transmission to be significant when compared with the direct transmission path (for example airborne transmission through the wall). Flanking transmission must be predicted by means of global models which take into account not only the insulation capacity of individual elements (i.e. walls or floors) but also the interaction between them and the global behaviour of the building.

A common approach in terms of prediction is to use the model proposed in the EN 12354 standard [1], which is based on the first order SEA formulation proposed in $[2,3]$. Among other data, it requires to know the vibration reduction index

$$
K_{i j}=\overline{D_{\nu, i j}}+10 \log _{10}\left(\frac{\ell_{i j}}{\sqrt{a_{i} a_{j}}}\right)
$$

where $\overline{D_{\nu, i j}}$ is the direction averaged vibration level difference, $\ell_{i j}$ is the length of the junction,

$$
a_{i}=\frac{2.2 \pi S_{i}}{c T_{i}} \sqrt{\frac{f_{r e f}}{f}}
$$

is the equivalent absorption length of the plate $i, S_{i}$ its surface, $c$ the speed of sound in the air, $f_{r e f}=1000 \mathrm{~Hz}$ is a reference frequency and $T_{i}$ the structural reverberation time of the wall $i$ that can be measured directly or calculated as $T_{i}=2.2 /\left(\eta_{\text {total }} f\right)$ (being $\eta_{\text {total }}$ the total loss factor).

$K_{i j}$ is the parameter that describes how the vibrations pass from one part of the building to another, typically through the junctions. Some of the problematic issues include the peaks and troughs in the vibration level differences due to low mode counts, and situation invariance due to low total loss factors in the laboratory and/or modal overlap factor (see discussion in [4]). The proper quantification of the flanking paths highly depends on the value of $K_{i j}$.

It can be obtained from experimental measurements of junctions in the laboratory [57]. However, it is time consuming, cumbersome and expensive. This is something to be done in a single junction but it is unrealistic to plan an experimental parametric analysis.

As an alternative to experiments, a common way to estimate $K_{i j}$ is by means of the formulas that are available in the Annex E of [1]. They cover: L, T and X homogeneous junctions, some variations including thin elastic layers between the junction parts and junctions of straight elements with different thickness. $K_{i j}$ can be easily obtained by the evaluation of a formula that depends on the mass ratio of the junction

$$
M=\log _{10}\left(\frac{m_{\perp}}{m_{i}}\right)
$$

$M$ is defined for the vibration transmission path from $i$ to $j, m_{i}$ is the mass per unit area of element $i$ and $m_{\perp}$ is the mass per unit surface of the orthogonal element.

Nevertheless, these empirical formulas have been mostly deduced from best-fit least-square curve of a series of measurements [2]. Furthermore, for a discussion concerning the problems related to the application of these empirical formulas, see [8]. On the one hand, this is good in the sense that reality was tested. But on the other hand, to be coherent with the SEA model in [2, 3], the data should represent first order paths. And it is highly probable that in situ measurements included high-order transmission paths. As shown in [9-11] it is not easy to define an error-free environment to compute or measure the vibration reduction index. In any case, there are evidences that if the prediction formulas in the Annex E [1] are compared with models of the isolated junction based on Finite Elements (FEM [12]), semi-analytical wave approach [8] or spectral elements (SFEM [13]) some differences are found. These differences 
are important in the following aspects:

- The formulas in the Annex E are frequency-independent and a single value of $K_{i j}$ is provided for the whole frequency range, while it is seen that $K_{i j}$ can be variable with frequency for some of the junctions and some of the transmission paths.

- The predicted values of $K_{i j}$ in junctions with mass ratio significantly different than one are quite different when computed with Annex E or some of the alternative models of the isolated junctions.

For these reasons, recent researches [8, 12-14] are trying to develop updated formulas that can be used to predict $K_{i j}$ with more details. Three different frequency ranges are defined: (i)low, from $50 \mathrm{~Hz}$ to $200 \mathrm{~Hz}$; (ii) mid, from $250 \mathrm{~Hz}$ to $1000 \mathrm{~Hz}$ and (iii)high, from $1250 \mathrm{~Hz}$ to $5000 \mathrm{~Hz}$. A single value of $K_{i j}$ is provided for each frequency zone: low, mid and high. It is calculated as the arithmetic mean of all third-octave band values. In addition, it was proposed in [12] to characterise the junction by means of the $P C$ variable ( $P C$ from Psi divided by $\mathrm{Chi}$ ) defined as

$$
P C=\log _{10}\left(\frac{\Psi}{\chi}\right)
$$

with

$$
\frac{\Psi}{\chi}=\sqrt[4]{\frac{m_{\perp} B_{\perp}^{3}}{m_{i} B_{i}^{3}}} \quad \text { with } B=\frac{E t^{3}}{12\left(1-\nu^{2}\right)}
$$

where $B$ is the bending stiffness per unit width $(\mathrm{Nm}), t$ the thickness, $E$ the Young modulus and the subscripts $i$ and $\perp$ refer to the source element and the orthogonal element respectively. The same Poisson's ratio $\nu=0.2$ for all the materials forming the junction is assumed according to the data provided in [15].

$\Psi / \chi$ includes information not only on the junction mass but also on the stiffness. The
$P C$ ratio is more adequate in order to characterise the junctions, especially when the vibration transmission is mainly caused by bending waves. It is, in fact, the ratio of characteristic moment impedances. As it was shown in [14], it reduces the scatter of data when bending transmission is dominant. It is possible that other parameters (i.e. combination of $\Psi / \chi$ and mass ratio) would reduce the scatter when the transmission is done by means of a combination of different wave types. Nevertheless, a proposal of a more adequate parameter is not a straightforward task.

The main goal of the current research is to extend the work presented in [13] to other junction types that are important for building industry but are not considered in the Annex E: H-shaped junctions, L or T junctions not forming a right angle, asymmetrical T-junctions, asymmetrical X-junctions. The data corresponding to the SFEM simulations in [14] are used in order to have reference values and regression curves for the standard L, $\mathrm{T}$ and $\mathrm{X}$-junctions. In fact, the present research is an extension of the work done with the SFEM model in [14] (no methodological change is introduced) to other junction typologies in order to have a more complete catalogue of formulas and gain knowledge on the behaviour of these less usual junctions not considered in [14] and the Annex E.

In the remainder of the document the method and model used is fast overviewed in Section 2, the results for each of the junction typologies mentioned above can be found in Section 3 and the conclusions are exposed in Section 5. Appendix A includes the tables with the coefficients obtained from the statistical analysis of the numerical results.

\section{Methodology}

The methodology used to study the new junctions is the same as described in [14]. The 
only difference is that, here, unpinned junctions are considered at low-frequencies. Pinning the junction is equivalent to assume that only bending waves are transmitted. The option here is to leave this aspect free and allow in-plane (longitudinal and transverse shear) waves to play their role if this is the case. The SFEM numerical model details as well as validations were presented in [13]. The numerical model, based on shell Spectral Finite Elements (SFEM [16-18]), is an efficient alternative to solve the elastodynamic problem in structures composed of extrusion symmetry shells in the frequency domain. The type of interpolation functions used makes this numerical model more adequate for high frequencies than FEM or other discretisation techniques. The reason is that no reduction in the element size is required when the frequency increases. This efficiency is important in order to perform the large number of simulations required for the parametric analysis, in order to derive general design rules and formulas, to cover a wide frequency range and reproduce the statistical/random nature of the physical phenomenon. An own implementation of the SFEM method is used. This is validated in single structural problems by comparing with the FEM software Code-Aster [19] and passing usual tests with available exact solution. A more general and large comparison was done in the framework of [14] where it was compared with wavebased models and $K_{i j}$ computations done with other FEM software. The model was also compared with $K_{i j}$ measurements on real junctions made in the laboratory [13].

The SFEM model deals with finite size junctions. It means that the proper modal behaviour of the junction is reproduced in each frequency range: a more or less random behaviour at low frequencies controlled by particular modes, and almost SEA behaviour at mid frequencies when the modal overlap increases. However, the final result is assumed to be independent on the par- ticular dimensions of the junctions (as the formulas in the Annex E are). With this purpose in mind and considering the large variability that input data and outputs in building acoustics can have (see for example $[20,21])$, a group of 27 junction dimensions are always averaged in order to obtain a single result, representative for all of them. The plate dimensions considered are shown in Table 1 , according to the sketch and notations in Fig. 1. The definition of which is the orthogonal element for each junction type and path in order to compute $\Psi / \chi$ in Eq. (5) is done in Table 3.

All the results presented here are limited to the case of point force excitation. Three different force positions in every plate are considered. Each position is considered independently for a total of three different simulations. Afterwards, the three results are averaged. In total, six force positions are needed to characterise a path because two different plates need to be excited. The positions are chosen in a quite random way but trying to be representative of all these: a point force acting on the central part, on the side, close and away of the junction. The criteria and restrictions on the excitation position described in ISO 10848-1 [22] are respected. The vibration fields are averaged all around the plate. A preliminary study on the influence of the excitation type and the average procedure was presented in [23]. Some variations exist but they are, on the one hand less important than the variations on $K_{i j}$ caused by other aspects like the dimensions or the uncertainties of the real junction details and properties. On the other hand they follow a quite general pattern and it seems possible to take them into account on the basis of the results presented here.

All the junctions in Fig. 1 are simply supported (displacements in the cross-section plane blocked but free rotation) at the three plate boundaries of each rectangular branch. The fourth plate boundary, the junction line, has 
no constrain but continuity in the displacements and rotations between all the branches is imposed. The displacements which are parallel to the junction line (orthogonal to the junction cross-section of Fig. 1) are not blocked.

A population of junctions is considered for each typology. This is generated with the material parameters of Table 2, considering: (i) homogeneous junctions made of concrete, aerated concrete blocks and calcium silicate blocks; (ii) junctions made of concrete floors (i.e. zones 2 and 5 in the H-junction) and other materials in the walls (i.e. zones 1,3 , 4 and 6 in the H-junction): aerated concrete blocks, bricks or dense aggregate blocks. The total damping is composed by the internal damping $\left(\eta_{\text {int }}\right)$ plus the boundary losses considered with $\eta_{\text {boundary }}=f^{-0.5}$. This is the option also chosen in [14] based on [24].

The thicknesses of each zone can be 0.1 , 0.2 and $0.3 \mathrm{~m}$ with a total of 9 possible combinations. Some more geometry detail of each junction typology will be specified when presenting the analysis and results. A range of mass ratios according to common junctions found in heavyweight buildings is therefore covered.

\subsection{Statistical analysis}

The population of $K_{i j}$ values obtained for each junction type and transmission path are approximated by means of the following equation

$K_{i j}=C_{0}+C_{1} P C+C_{2} P C^{2}+C_{3} P C^{3}+C_{d} d+C_{\theta} \theta$

Coefficients $C_{0}, C_{1}, C_{2}$ and $C_{3}$ are considered in all the junction types while $C_{d}$ is used only for the H-junction and $C_{\theta}$ for those junctions that have some part not forming a rightangle. $d$ is the separation between the leaves of the H-junction in cm (see Fig. 1(b)) and $\theta$ is the angle that can be seen in Fig. 1(a) and (e).

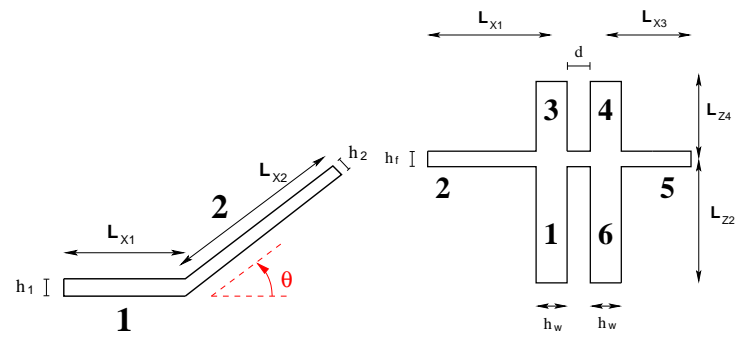

(a)

(b)

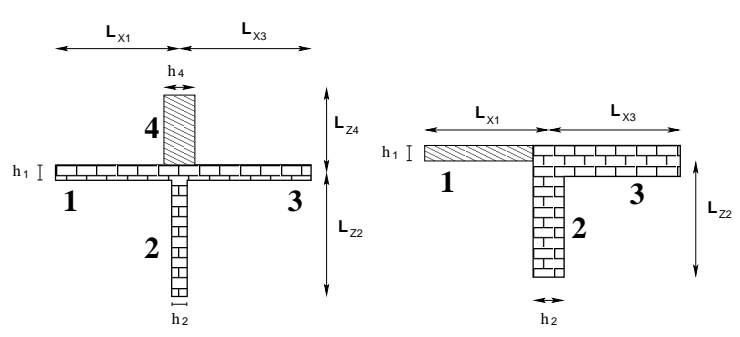

(c)

(d)

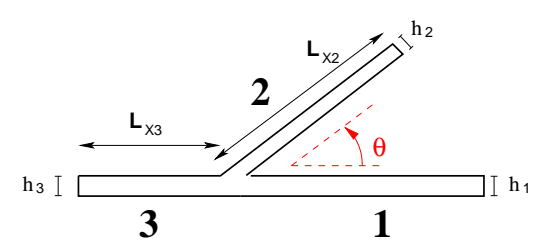

(e)

Figure 1: Sketch and notation of the junctions: (a)L-junction with variable angle $\theta$; (b) $\mathrm{H}$-junction $\left(t_{1} \equiv t_{6} \equiv t_{3} \equiv t_{4}\right.$ and $t_{2} \equiv$ $\left.t_{5}\right)$; (c) Asymmetrical X-junction $\left(t_{1} \equiv t_{2} \equiv\right.$ $\left.t_{3}\right)$; (d) Asymmetrical T-junction $\left(t_{2} \equiv t_{3}\right)$; (e) T-junction with variable angle $\theta\left(t_{1} \equiv t_{3}\right)$.

Other functional dependences on the separation between leaves (i.e. $\sqrt{d}$ ) or the angle between the junction parts (i.e. $\cos (\theta)$ ) have been tested. However, they lead to statistical descriptions of the results of equivalent quality in terms of standard error. In view of the very small or null improvement, the option was to keep Eq. (6) as simple as possible.

$K_{i j}$ is chosen here as the independent variable because it is the output directly obtained in finite dimension junctions (i.e. finite / spectral element simulations or laboratory measurements). The alternative was to use the transmission loss $T L_{i j}$ as done in [14].

$$
T L_{i j}=-10 \log _{10}\left(\gamma_{i j}\right)
$$




\begin{tabular}{lll}
\hline Parameter & Symbol & Value \\
\hline Lengths in extrusion direction & $L_{y}$ & $4.0,5.0,6.0 \mathrm{~m}$ \\
Lengths for the floors $(\mathrm{X}, \mathrm{T}, \mathrm{L}$ and $\mathrm{H})$ & $L_{x}$ & $3.5,4.5,5.5 \mathrm{~m}$ \\
Height for the wall $(\mathrm{X}, \mathrm{T}$ and $\mathrm{H})$ & $L_{z}$ & $2.5 \mathrm{~m}$ \\
\hline
\end{tabular}

Table 1: Geometrical properties of the plates.

\begin{tabular}{lcccc}
\hline Material & $\begin{array}{c}\rho_{v} \\
\left(\mathrm{~kg} / \mathrm{m}^{3}\right)\end{array}$ & $\nu$ & $\begin{array}{c}E \\
(\mathrm{GPa})\end{array}$ & $\eta_{\text {int }}$ \\
\hline Concrete & 2200 & 0.2 & 30.5 & 0.005 \\
Aerated1 & 400 & 0.2 & 1.39 & 0.0125 \\
Aerated2 & 800 & 0.2 & 2.77 & 0.0125 \\
Dense & 2000 & 0.2 & 19.7 & 0.01 \\
Bricks & 1750 & 0.2 & 12.2 & 0.01 \\
Calcium & 1800 & 0.2 & 10.8 & 0.01 \\
\hline
\end{tabular}

Table 2: Material properties (frequency independent) of the parametric analysis. Same materials as [8] have been considered. 'Aerated' means Aerated concrete blocks (of two different types, 1 and 2). 'Dense' means Dense aggregate blocks and 'Calcium' is used for Calcium silicate blocks. $\rho_{v}$ is the volumetric density.

with the angular-average transmission coefficient, $\gamma_{i j}$, for bending wave transmission from plate $i$ to plate $j$ is related to the vibration reduction index by

$$
-10 \log _{10}\left(\gamma_{i j}\right)=K_{i j}-5 \log _{10}\left(\frac{f_{c, j}}{f_{\text {ref }}}\right)
$$

and $f_{c, j}$ the critical frequency of element $j$ in Hz. It was shown in [14] that using $T L_{i j}$ reduces the scatter of data points (simulation outputs), especially in the junctions where bending transmission is dominant. It was also a good choice in [14] because data generated with wave-based methods (infinite junctions) and FEM or SFEM models (finite-dimensions junctions) needed to be combined and compared somehow. However, it is not the natural option here where only data generated by means of SFEM is analysed. In addition, the relationship between $K_{i j}$ and $\gamma_{i j}$ Eq. (8) is not exact. It would introduce another possible source of data points scatter which is better to avoid.

A multi linear regression of the data points is carried out using the free software for statistical analysis of sampled data PSPP [25]. The coefficients that are the output of the analysis are listed in the tables of Appendix A. Tables 9, 10 and 11 are based on the SFEM simulations on the standard junctions done in [14] and are mainly considered to have a reference and for the sake of completeness of the collection/catalogue. The differences found between all the models in [14] were not large. Consequently a regression formula for $K_{i j}$ based on the full data set are not expected to differ very much around $\Psi / \chi=1$ from the ones proposed in Tables 9, 10 and 11.

In all the figures, the curves obtained from the regression analysis are labelled with the letter ' $\mathrm{R}$ '.

\section{Results}

\subsection{H-shaped junctions}

Few studies on the vibration reduction index of H-shaped junctions can be found. See [12] that contains $K_{i j}$ expressions for the mid frequency range obtained from FEM simulations and [26, 27] for a wave-based model covering several conceptual aspects of the $\mathrm{H}-$ junction and double T-junction behaviour.

The sketch of the junction and the notation for each part is shown in Fig. 1(b). In addition to the comments of Section 2, it must 


\begin{tabular}{|c|c|c|c|c|c|c|c|}
\hline Junction & Path & $\perp$ & $i$ & Junction & Path & $\perp$ & $i$ \\
\hline \multirow[b]{2}{*}{$\mathrm{H}$} & $2-5,2-4,2-3$ & $3 \equiv 4 \equiv 1 \equiv 6$ & $2 \equiv 5$ & \multirow{2}{*}{$\mathrm{T}$ angle } & $1-2$ & 2 & $1 \equiv 3$ \\
\hline & $3-1,3-4$ & $2 \equiv 5$ & $3 \equiv 4 \equiv 1 \equiv 6$ & & $1-3$ & 2 & $1 \equiv 3$ \\
\hline Asym. X & $1-3, \overline{2}-4$ & 4 & $1 \equiv 3 \equiv 2$ & Asvm $\mathrm{T}$ & $\overline{1}-\overline{2}, \overline{2}-$ & $2 \equiv \overline{3}$ & 1 \\
\hline & $1-4,1-2$ & $1 \equiv 3 \equiv 2$ & 4 & & $1-3$ & $2 \equiv 3$ & 1 \\
\hline
\end{tabular}

Table 3: Definition of the orthogonal element to compute $\Psi / \chi$ in Eq. (5) for all the junction types in Fig. 1.

be noted that walls $1,3,4$ and 6 have always the same material properties and thickness. The same comment is valid for the floors 2 and 5. Four different separations between the internal faces of the walls $3-4$ and $1-6$ are considered: $d=0.05,0.1,0.2$ and $0.3 \mathrm{~m}$.

The $K_{i j}$ of five different paths are computed. Only some illustrative results are shown in Figs. 2, 3 and 4. The results for the $\mathrm{H}$ junction are compared with the $K_{i j}$ values for the standard X-junction (Eq. (6) with the coefficients in Table 10). Figs. 2 and 3 contain the data for the more characteristic paths in the $\mathrm{H}$-junction which are affected by the separation between leaves: $K_{25}$ and $K_{24}$ respectively. While Fig. 4 shows results in the mid frequency range for the other three studied paths: $K_{23}, K_{31}$ and $K_{34}$. These mid frequency results are representative because, even if numerical values do vary with frequency, not many differences concerning the shape of the function and general trends (distribution of data, quality of the statistical approximation,...) were found due to the variation of frequency in the three mentioned paths.

Fig. 2 shows the straight transmission between the floors 2 and 5 , in the direction perpendicular to the double wall. $K_{i j}$ decreases with frequency. There is also a dependence on the separation between the leaves of the double wall $(d)$. It causes larger variations of $K_{i j}$ for junctions with $\Psi / \chi>1$ (this is not the most usual junction type when 2 and 5 are the floors). Even if a global trend can be noted $\left(K_{i j}\right.$ tends to be larger for the junc- tions with larger separation $d$ ), a large spread also exist. This makes it difficult to adjust a regression curve according to Eq. (6). The standard error in Table 4 for the path $2-5$ is quite large which makes it difficult to use the proposed formulas for this path with acceptable precision.

The H-junction results are compared with two different predictions of the standard Xjunction. On the one hand a single $\mathrm{X}$-junction with the floors 2 and 5 and the walls 1 and 3 ('X (3)'). On the other hand a X-junction where the walls are considered to have double thickness, which is equivalent to put together the leaves $1+6$ and $3+4$ (' $\left.\mathrm{X}(3+4)^{\prime}\right)$. Doubling the thickness is equivalent to considering the two leaves acting as a single leaf. It is important in terms of the stiffness, which is computed with an effective thickness $2 t$ instead of making the addition of the stiffnesses of the isolated leaves (that would probably be an intermediate option). At mid and high frequencies, the results are between the two curves: single and double X-junction. However, at low frequencies $K_{i j}$ can be even larger than the predictions done with a double $\mathrm{X}$-junction (the closest results to the double $\mathrm{X}$-junction are those for $d=0.1 \mathrm{~m}$ ).

Fig. 3 is illustrative of the right-angle transmission between the floor 2 and the second wall 4 . This transmission path is important for those double walls that have structural continuity under or over their core. In that situation the vibrations can bypass the insulating cavity and produce sound radiation in the contiguous rooms by direct excitation of 


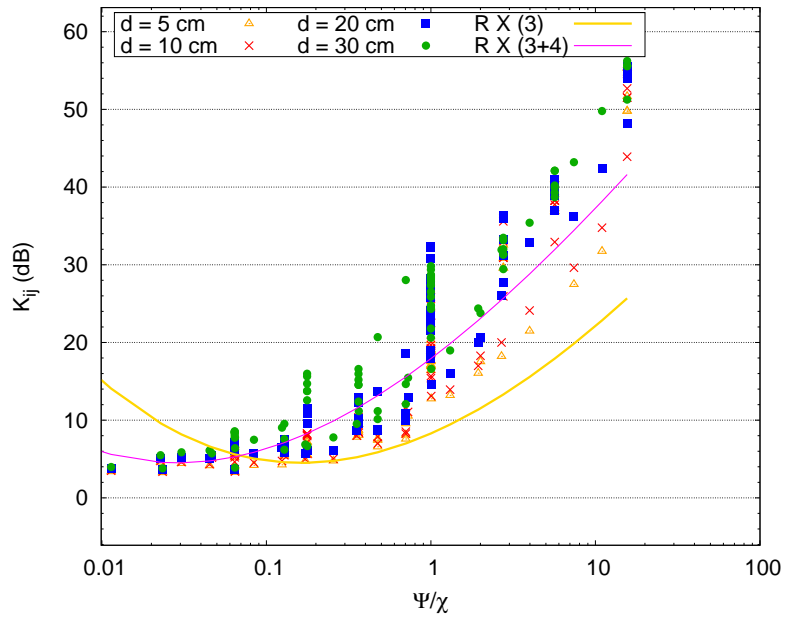

(a)

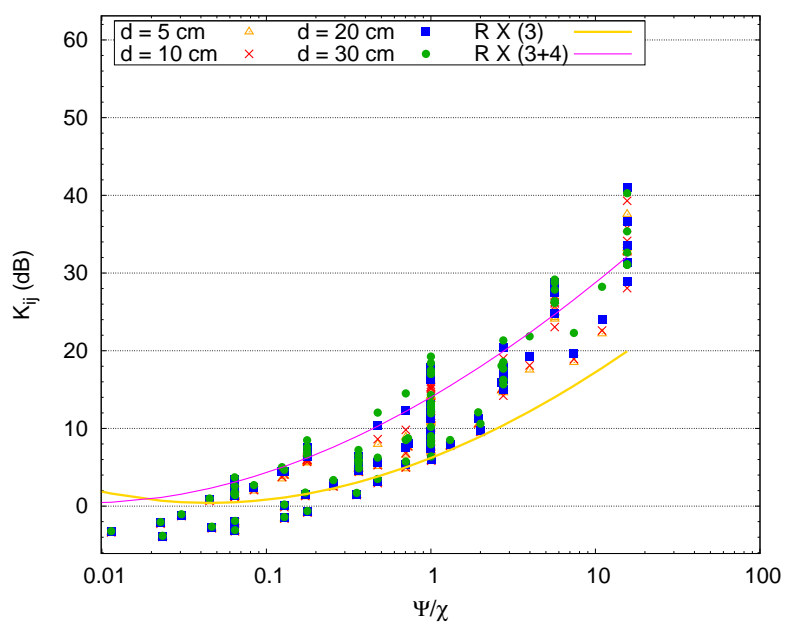

(b)

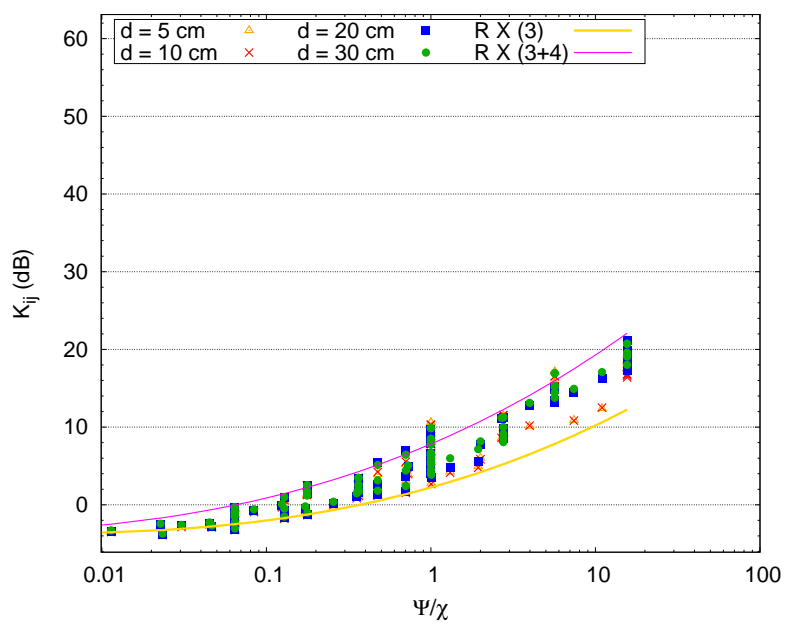

(c)

Figure 2: H-shaped junction, in-line transmission $\left(K_{25}\right)$. Comparisons with the expressions of the standard X-shaped junction. Three frequency ranges are shown: (a)Low; (b)Mid; c)High. the leave 4 (see for example [28]).

In that case, the separation between the leaves of the double wall $(d)$ is also relevant and we can see in Fig. 3 how the data points are more or less ordered from $d=0.05 \mathrm{~m}$ (the ones with smaller $K_{i j}$ ) to $d=0.3 \mathrm{~m}$ (the ones with larger $K_{i j}$ ). However, the spread is large and a regression with Eq. (6) leads to a quite poor adjustment with large standard errors. This effect is even worse at low frequencies and slightly better at high frequencies. In both cases the plot of data looks similar to Fig. 3 with the mentioned difference in the spread.

In almost all the cases, the vibration insulation is larger than for an X-junction composed of parts 2, 5, 4 and 6 (with a single wall). This is logical because the wall 3 represents a barrier for the vibrations that go from part 2 to 4 and vice versa. This effect is more important for the junctions with $\Psi / \chi>1$ (i.e. with heavier or stiffer 3 and 4). A better lower bound, at least for the zone $\Psi / \chi>1$, is obtained if an orthogonal wall with double thickness is used in the X-shaped formula $\left(' \mathrm{X}(3+4)^{\prime}\right)$. This is even more right for the smallest separation distance. For $\Psi / \chi<1$ to consider the single $\mathrm{X}$-junction as lower bound could be more accurate.

The $K_{24}$ curve is not symmetrical with respect to the vertical axis $\Psi / \chi=1$. This can be explained by the different physical phenomena that control the vibration transmission for $\Psi / \chi>1$ and $\Psi / \chi<1$. For $\Psi / \chi>1$ the main aspect that controls the vibration transmission is the blocking role that the wall 3 plays in the path $2-4$. This phenomenon is in addition to the fact that the properties of the floors 2, 5 and walls 3,4 are also different which does not help the transmission of vibrations. In the limit, for very high values of $\Psi / \chi$ the vibration transmission must tend to infinite. But this increase is with larger slope than for the equivalent $\mathrm{X}$-junction because in the $\mathrm{H}$-junction we have the two aspects (change of properties and wall block- 
ing) collaborating in the same direction while in the equivalent $\mathrm{X}$-junction only one (change of properties). For $\Psi / \chi<1$ the blocking role of wall 3 tends to be marginal and the predominant aspect in the vibration isolation is the difference in the properties between parts. In the limit for very small $\Psi / \chi$ the vibration isolation increases more or less following a line in all cases. The slope of this line is very similar to the slope of a line that would adjust the curve (' $\mathrm{X}(3+4)$ ') for $\Psi / \chi<0.1$. This means that the main phenomenon is the difference in the properties between parts and the wall blocking effect tends to be marginal. It must be also noted that due to the distribution of geometric and material properties assumed (same properties in zones 2 and 5; and in zones 1, 3, 4, and 6 ) the path 2-4 does not show symmetry.

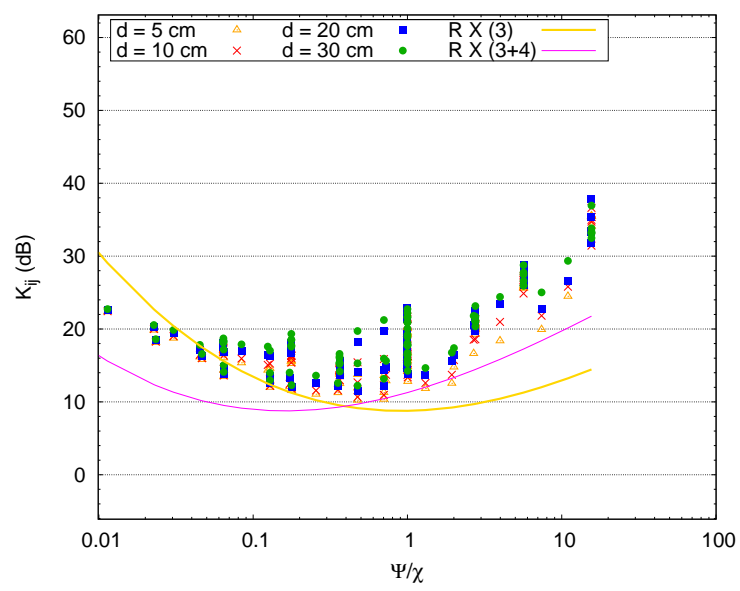

Figure 3: H-shaped junction, right-angle transmission to the second separating wall $\left(K_{24}\right)$, mid-frequency.

The paths $2-5$ and $2-4$ are the ones that make the $\mathrm{H}$-junction differ more from the $\mathrm{X}$ junction. Also the separation between the leaves 3 and 4 is more relevant in these two paths causing a large spread of the vibration reduction index values. The parameter $d$ also causes an important spread of $K_{i j}$ values in the path 3-4 (see Fig. 4(c)) but not so much in two of the other studied paths $(2-3$ and $3-1$ in Fig. 4(a) and (b)).

In the transmission paths $2-3$ (see Fig. 4(a)) and 3-1 (see Fig. 4(b)) the response of the Hjunction is very similar to those of the standard X-junction.

\section{$3.2 \quad$ Non-orthogonal junctions}

Some junctions where the plates form an angle different from the right-angle are considered. L-junction (for the sketch and notation, see Fig. 1(a)) and T-junction (see Fig. 1(e)) are quite representative to derive general conclusions from their results. In all the figures of this section, the data points are shown with the proposed regression formulas. They are based on Eq. (6) with the coefficients in: Table 5 (L-junction with variable angle), Table 11 (right-angle L-junction), Table 6 (Tjunction with variable angle), Table 9 (rightangle T-junction). All the tables can be found in Appendix A. The specific regression formulas for $\mathrm{L}$ and $\mathrm{T}$ junctions with variable angle are compared with the formulas for the standard $\mathrm{L}$ and $\mathrm{T}$ junctions (with the plates forming a right-angle). A discussion on the meaning of all variants of Table 5 is done in Section 4.1 (in the current Section, the rows with the label 'Non-symmetric data and Nonsymmetric formula' are considered).

\subsubsection{L-junctions}

Some representative results for the L-junction are shown in Fig. 5 (when the angle $\theta$ is smaller or equal than $90^{\circ}$ ) and Fig. 6(when the angle $\theta$ is larger or equal than $90^{\circ}$ ). The main conclusion is that $K_{i j}$ takes the maximum value for the orthogonal (usual) L-junction. And $K_{i j}$ decreases when the angle $\theta$ tends to $0^{\circ}$ or $180^{\circ}$.

A rule of thumb in order to approximate the influence of the angle variation is

$$
\Delta K_{i j} \simeq-0.1|\Delta \theta|
$$




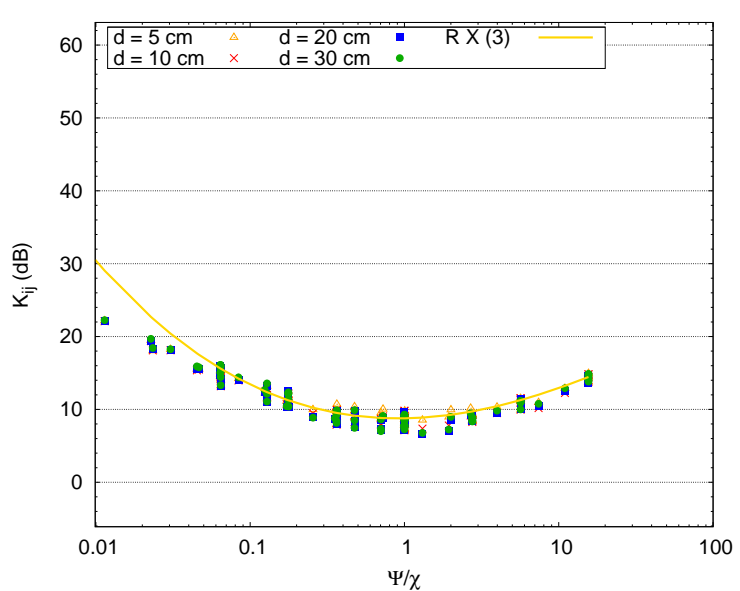

(a)

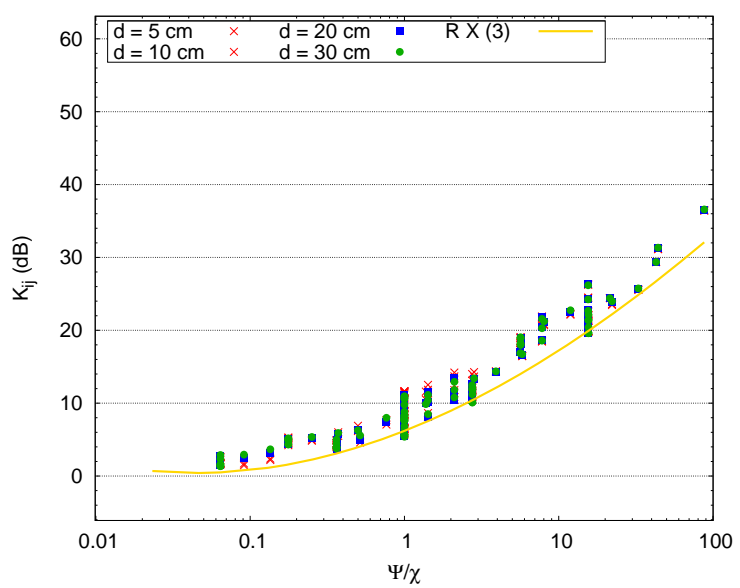

(b)

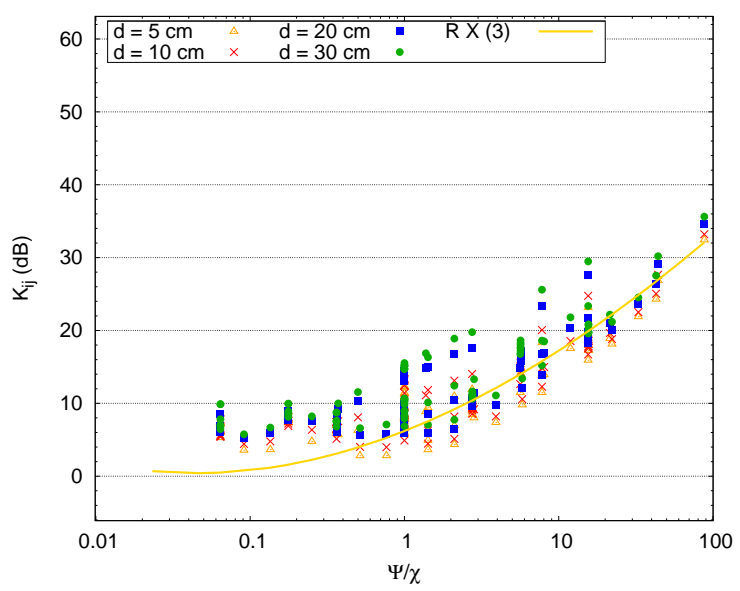

(c)

Figure 4: H-shaped junction, results in the mid-frequency range. Vibration reduction index for several transmission paths:(a) $K_{23}$; (b) $K_{31}$; (c) $K_{34}$. where $\Delta K_{i j}$ is the decrease in the vibration reduction index with respect to the rightangle junction $\left(90^{\circ}\right.$, with regression coefficients at Table 11). This can be more precisely done through the regression coefficient $C_{\theta}$ in Table 5. This angle-dependency is clearer at mid and high frequencies when almost all the data points tend to be aligned with the proposed regression curve. The worst situation in terms of spread of data points is found at low frequencies when $\theta$ is smaller or equal than $90^{\circ}$ (see Fig. 5(a)). It is also reflected in the regression analysis with standard error larger than $3 \mathrm{~dB}$ (see Table 5, using the coefficients for 'Non-symmetric data and Non-symmetric formula'). It makes no sense to plot and use the regression curves in Fig. 5(a) because the differences are too large. The situation would probably be clearer if the L-junction was pinned. In that case only bending waves would be interacting (and the parameter $\Psi / \chi$ describes especially well this situation).

Fig. 5 includes also the $K_{i j}$ formula proposed in the [1] for the straight junction of different thickness and material: $K_{i j}=5 M-$ $5 \mathrm{~dB}$. It seems a quite good lower bound in the limit when $\theta$ tends to $0^{\circ}$.

The statistical analysis also shows some slight asymmetry (in the shape of the curves) between the cases with $\theta \leq 90^{\circ}$ and $\theta \geq 90^{\circ}$. This is seen through the values of the coefficient $C_{2}$ which are a bit larger when $\theta \geq 90^{\circ}$. Another important aspect is to see the limit of Eq. (6) when $\theta=90^{\circ}$. It is compared with the curve that uses the coefficients for the standard L-junction in Table 11 (labelled with ' $R$ L'). The second option always fits better with the data points of the junctions with $\theta=90^{\circ}$ because it is generated with this purpose. However the agreement is acceptable, especially at mid and high frequencies. The differences are because the general formula (with variable $\theta$ ) needs to fit all data points with different angle and each of them exhibits a slightly different concavity. 
The cases with $\theta$ much larger than $90^{\circ}$ would behave more like an U-junction (with short middle plate) instead of an L-junction. They are not very common, especially for dwellings.

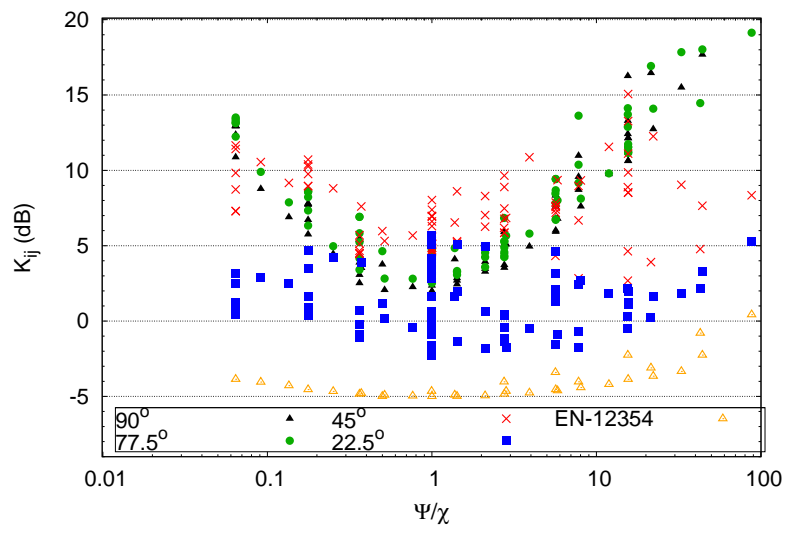

(a) Low, $\theta \leq 90$

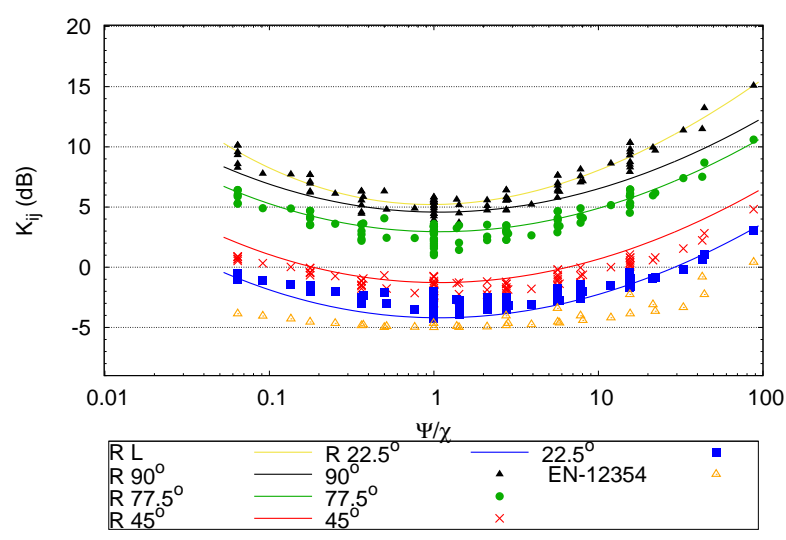

(b) High, $\theta \leq 90$

Figure 5: Vibration reduction index $K_{i j}$ for the L-shaped junction with angle $\theta$ smaller or equal than $90^{\circ}$ for several frequency ranges: (a) Low; (b) High. The label 'EN-12354' identifies the values obtained with the $K_{i j}$ formula proposed in the [1] for the straight junction of different thickness and material.

\subsubsection{T-junctions}

The main difference here with respect to Section 3.2.1 is that two different transmission paths can be studied: through the angle $\left(K_{12}\right.$, also existent in the L-junction) and through

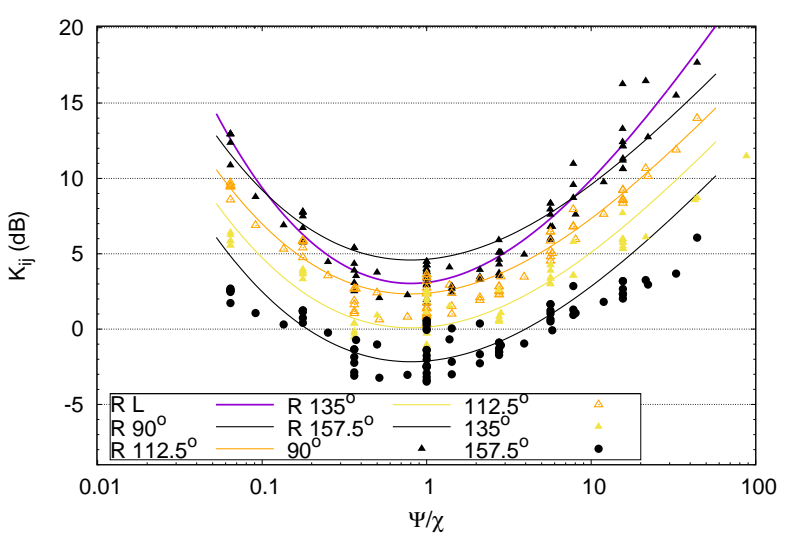

(a) Low, $\theta \geq 90$

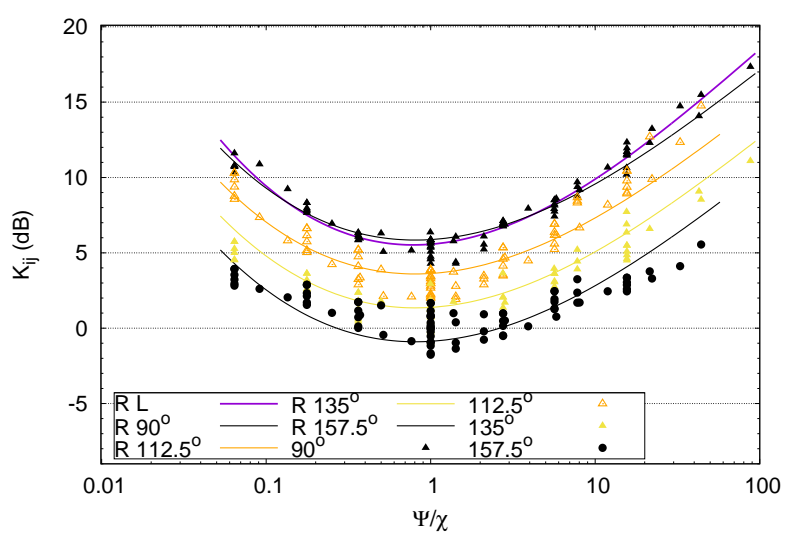

(b) Mid, $\theta \geq 90$

Figure 6: Vibration reduction index $K_{i j}$ for the L-shaped junction with angle $\theta$ larger or equal than $90^{\circ}$ for several frequency ranges: (a) Low; (b) Mid.

the straight section $\left(K_{13}\right.$, specific of the Tjunction).

Fig. 7 shows illustrative results of the vibration reduction index $K_{13}$ in the transmission through the straight section. The junction behaviour is conceptually very similar for both cases: $\theta \leq 90^{\circ}$ and $\theta \geq 90^{\circ}$. At low frequencies the spread of data points is large (see the standard errors of the regression in Table 6 which are around $3 \mathrm{~dB}$ ). This makes it sometimes even difficult to establish a proper classification of the $K_{13}$ values in terms of the junction angle. The gradation and quality of statistical approximation is much better at mid frequencies. At high 
frequencies the influence of $\theta$ on the vibration reduction index $K_{13}$ is almost null and all the junctions with the same value of $\Psi / \chi$ have similar value of $K_{13}$ (see Fig. 7 (b)).

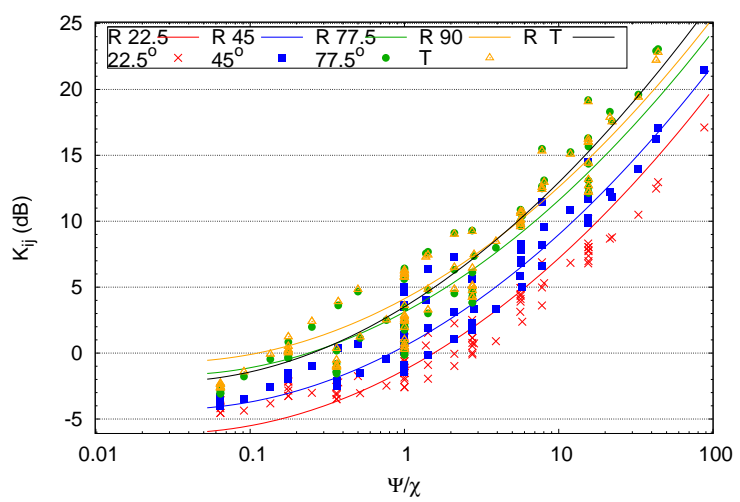

(a) Mid, $\theta \leq 90^{\circ}$

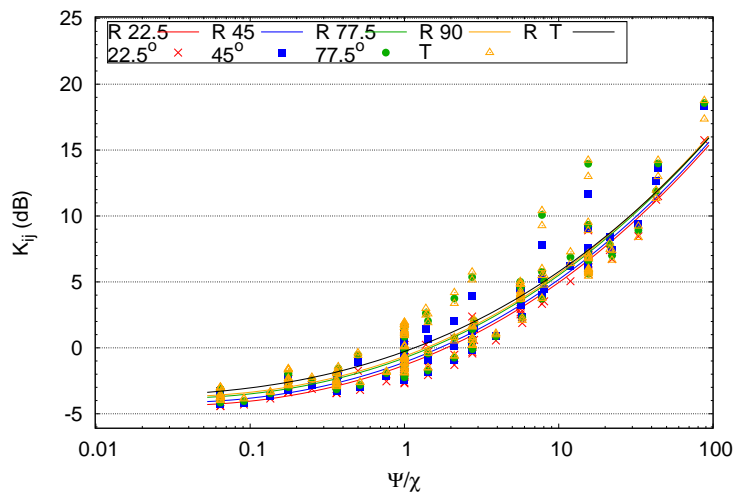

(b) High, $\theta \leq 90^{\circ}$

Figure 7: T-shaped junction with variable junction angle, straight transmission $\left(K_{13}\right)$. Two different frequency ranges: (a) mid; (b) high.

Fig. 8 shows illustrative results of $K_{12}$ in the transmission through the angle. In that case it is more clearly seen the influence of the junction angle $\theta$. The statistical analysis of the data leads to better regression formulas. Except for the low-frequency response for $\theta \leq$ $90^{\circ}$.

Eq. (9) approximates quite well the angle influence also for the T-junctions (see Figs. 7 and 8), except for the transmission through the straight section at high frequencies where this effect can be neglected (Fig. 7(b)).

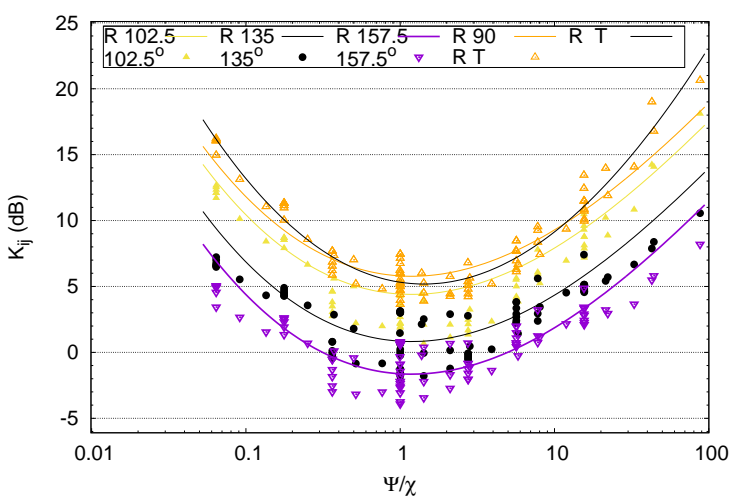

(a) Low, $\theta \geq 90^{\circ}$

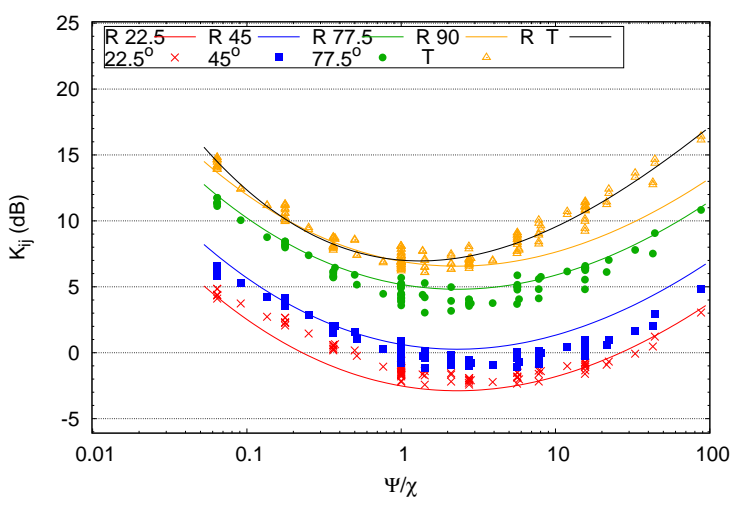

(b) High, $\theta \leq 90^{\circ}$

Figure 8: T-shaped junction with variable junction angle, transmission through the angle $\left(K_{12}\right)$.

\subsection{Asymmetrical junctions}

The formulas for the $\mathrm{T}$ and $\mathrm{X}$ junctions in the Annex E as well as in recent researches $[8,12,13]$ consider only symmetrical junctions with respect to mass per unit area. According to the notations in Fig. 1(c) and (d), it means that parts 1 and 3 have always the same thickness and mechanical properties while part 2 can freely vary its properties. In addition, for the $\mathrm{X}$-junction the properties of part 4 must be coincident with the ones in part 2 . All these keep the symmetry of the junction and the mass ratio $(M)$ or the $\Psi / \chi$ parameter establishes the relationship between orthogonal elements.

However, real buildings can have junc- 
tions where this symmetry is lost. This situation is not considered in Annex E and rarely in the literature. A procedure based on the hypothesis that the difference between the sound reduction index of a flanking path and the vibration reduction index is the same for symmetric and for asymmetric junctions was proposed in [29, 30]. This helped in order to obtain $K_{i j}$ curves by means of statistical fitting of many measured flanking paths. A wave based model was formulated in [31] to deal with asymmetrical $\mathrm{X}$-junctions. The model considered bending waves and the transmission loss, taking into account the transmission direction, was computed.

Here it is considered that in the asymmetrical $\mathrm{T}$-junction the thickness and mechanical properties of parts 2 and 3 are the same and independent of part 1 (this is a small simplification because the real asymmetrical junction would have three different properties). In the asymmetrical $\mathrm{X}$-junction only the part 4 will have different properties than the other three parts, that will be made of the same material with equal thickness.

$\Psi / \chi$ is computed as the properties of part 4 divided by the properties of parts 1, 2 and 3, for the asymmetrical X-junction (see Fig. 1 (c)). And for the T-junction $\Psi / \chi$ is computed as the properties of part 2 and 3 divided by the properties of part 1 (see Fig. 1 (d)).

Fig. 9(a) shows the straight transmission $(1-3)$ in the T-junction. When it is symmetrical, $K_{i j}$ increases if the orthogonal part (2) is heavier or stiffer than the others (1 and 3 ). Otherwise $(\Psi / \chi<1), K_{i j}$ remains more or less constant. On the contrary, for the asymmetrical T-junction, $K_{i j}$ increases in both situations (large and small $\Psi / \chi$ ) and has the minimum value around $\Psi / \chi \simeq 1$. Two aspects could explain this behaviour. On the one hand, for $\Psi / \chi>1$, the heavier orthogonal part of the junction makes it difficult to transmit vibrations in the direction $1-3$. On the other hand, the different properties of parts 1 and 3 represents an impedance mismatch which implies extra difficulty in order to transmit the vibrations between them. And this explains the increase of $K_{i j}$ even for $\Psi / \chi<1$.

The right-angle transmission between the parts 1 and 2 in the asymmetrical T-junction, see Fig. 9(b), seems to suffer a shifting in the negative direction of the $\Psi / \chi$ parameter. This implies that $K_{i j}$ is larger in the zone where $\Psi / \chi>1$ and smaller in the zone where $\Psi / \chi<1$. The minimum value is located around $\Psi / \chi=0.5$. And for the path $2-3$ a very asymmetrical (with respect to the vertical axis $\Psi / \chi=1$ ) behaviour of the $K_{i j}$ curve is observed. This makes sense because for $\Psi / \chi>1$ the path $2-3$ behaves almost like an $\mathrm{L}$ junction with constant value of $\Psi / \chi=1$ (this is most true for mid and high frequencies). Since the part 1 is much less heavy and stiff when $\Psi / \chi>1$ it has a small influence. On the contrary when $\Psi / \chi<1$ the part 1 of the junction tends to control all the transmission between 2 and 3, which rapidly becomes a path with much less transmission with respect to the path $1-2$ or the standard T-junction. At mid and high frequencies and $\Psi / \chi<1$, the transmission in the path $2-3$ is almost the same as for the standard T-junction.

The right-angle transmission from 1 to 4 in the the X-junction, see Fig. 10(b), has a very similar behaviour to the T-junction (Fig. 9(b)). The comments for the T-junction are still valid here, especially for $\Psi / \chi<1$. The differences between symmetric and asymmetric $\mathrm{X}$-junctions in the range $\Psi / \chi>1$ are less than for the T-junction. The right-angle transmission through the path $1-2$ is similar to the path $2-3$ in the assymetrical T-junction. There is assymetrical behaviour with respect to the vertical axis $\Psi / \chi=1$. For $\Psi / \chi>1$ the junctions tend to behave like a standard T-junction with $\Psi / \chi=1$ because the part 4 is much less heavy and stiff than the parts 1,2 and 3. On the contrary, 


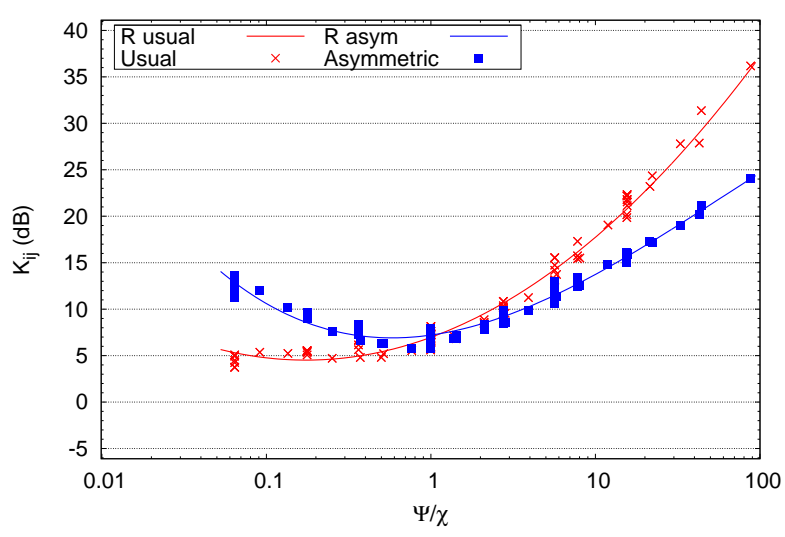

(a)

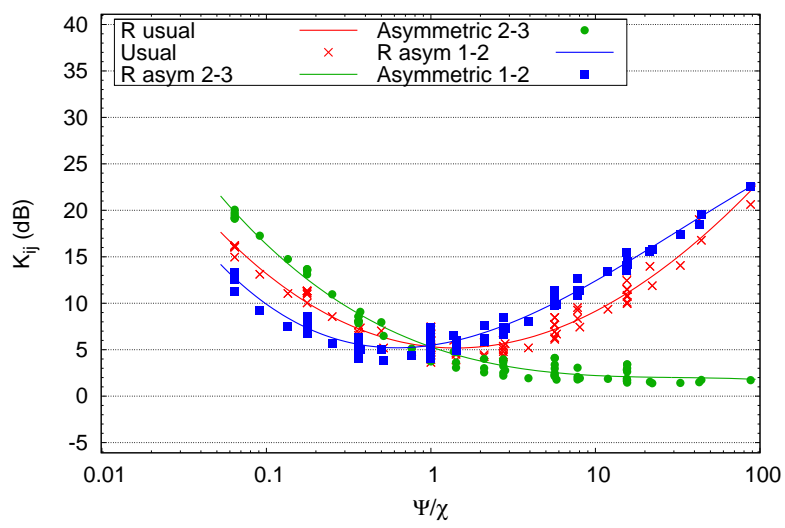

(b)

Figure 9: Comparison of the vibration reduction index $K_{i j}$ for the usual and asymmetrical $\mathrm{T}$-junction in the low-frequency range $(50-200 \mathrm{~Hz})$ : (a) Straight transmission; (b) Right-angle transmission $(1-2$ and $2-3)$.

for $\Psi / \chi<1$ the part 4 makes difficult the vibration transmission between parts 1 and 2 .

The other paths studied here for the Xjunction are the straight transmissions $1-3$ (between elements having the same properties) and 2-4 (between elements having different properties). The path $1-3$ is conceptually different to the path $1-3$ in the asymmetrical T-junction because there is no thickness or material change in the transmission direction but only in one of the perpendicular elements. For $\Psi / \chi<1$ the part 4 is much less heavy and stiff than the others and the junction behaves like a standard T-junction with $\Psi / \chi=1$. For this reason the path $1-3$ in the assymetric X-junction is almost constant (horizontal) for $\Psi / \chi<1$. On the contrary for $\Psi / \chi>1$, part 4 makes it difficult the vibration transmission from 1 to 3 and the isolation increases. $K_{i j}$ is smaller than for the standard $\mathrm{X}$-junction here because the part 2 in the assymetric X-junction is less heavy and stiff (in the standard, the parts 2 and 4 have the same properties).

The transmission path $2-4$ has a minimum value of $K_{i j}$ around $P C=1$ but increases on the other two sides. The increase is more important for $\Psi / \chi<1$ because in addition to the dissimilarity of parts 2 and 4 , the blocking role of parts 1 and 3 is important for $\Psi / \chi<1$ and can be almost neglected for $\Psi / \chi>1$.

Fig. 10 includes diamonds (with the label ' $\mathrm{R}$ Usual $\left.(2+4 / 2)^{\prime}\right)$. They represent data generated with the regression formula for the standard X-junction ' $\mathrm{R}$ usual' (coefficients in the Table 10) but considering averaged properties between walls 2 and 4. All properties (thickness, Young modulus, density) are averaged before the computation of $P C$. The points show some dispersion because $P C$ is evaluated for exactly the same junctions considered in the analysis (i.e. the regression formula is not evaluated for a continuous set of $P C$ values but for the $P C$ values computed for every junction). The good agreement with the asymmetric data suggest that this path 1-3 (straight transmission) in the asymmetrical X-junction can be properly approximated with the standard formula but using averaged input parameters.

Figs. 9 and 10 are representative of the three frequency ranges. For a more detailed quantification of $K_{i j}$ see Tables 7 and 8 .

In general, a proper study of these junctions is required and the use of models and formulas for the standard symmetrical ones can lead to considerable errors. This has been quantified for the $\mathrm{X}$-junction. Table 8 


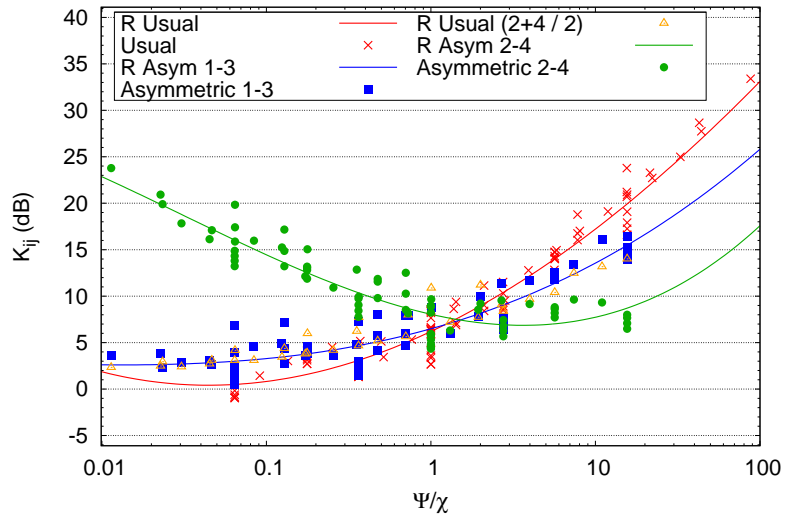

(a)

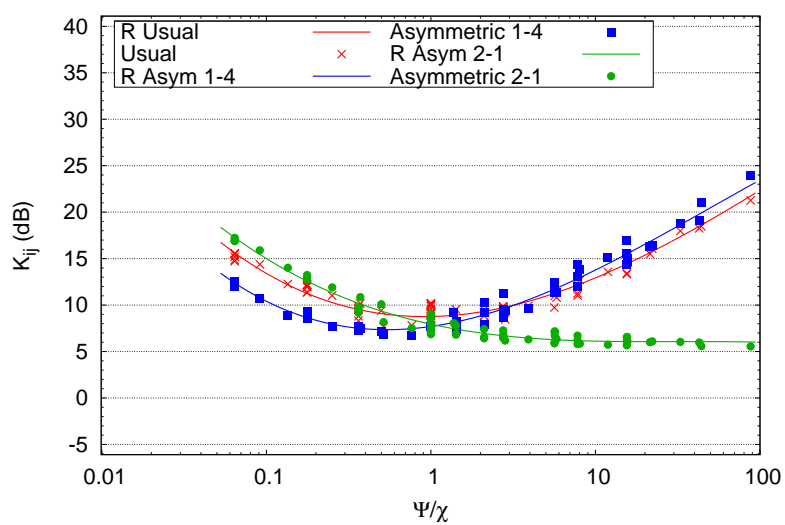

(b)

Figure 10: Comparison of the vibration reduction index $K_{i j}$ for the usual and asymmetrical a X-junction in the mid-frequency range $(250-1000 \mathrm{~Hz})$ : (a) Straight transmission $(1-3)$; (b) Right-angle transmission $(1-4)$. In (a), the diamonds ' $R$ Usual $(2+4 / 2)$ ' are data generated with the regression formula for the standard $\mathrm{X}$-junction ' $\mathrm{R}$ usual' but considering averaged properties between walls 2 and 4 .

shows two measures of standard error. On the one hand, the error due to the statistical analysis of the data for the asymmetrical X-junction. This is a measure of the difference between the proposed statistical formula and the available data (this is shown in all the tables). On the other hand, the mean differences found when the asymmetrical $\mathrm{X}$-junction data are compared with the values predicted by means of the regression formula of the standard X-junction (coefficients in Table 10). In both cases the standard error is computed as

$$
\text { std. Error }=\sqrt{\frac{1}{n} \sum_{r=1}^{n}\left(K_{i j}^{S}-K_{i j}^{P}\right)^{2}}
$$

where $n$ is the number of data points obtained with the SFEM simulations, $K_{i j}^{S}$ is the vibration reduction index computed in the simulations and $K_{i j}^{P}$ is the vibration reduction index predicted with the regression formulas. The error when using the regression formula for the standard $\mathrm{X}$-junction is always larger than the error found when the specific formula for the asymmetrical $\mathrm{X}$-junction is considered. The differences in the value of the standard error vary between 1 and 2.7 dB.

\section{Discussion}

\subsection{Symmetrisation of data}

Some of the junctions and paths involved in the analysis need to have symmetry due to the definition of $K_{i j}$ in Eq. (1): L-junctions at any angle, the path $1-2$ in symmetric Xjunctions (but note that T-junctions in the path $1-2$ do not need to respect the symmetry because the junction with $\Psi / \chi$ and $(\Psi / \chi)^{-1}$ are not the same junction). This means that $K_{i j}$ must be symmetric with respect to a vertical axis where $\Psi / \chi=1$. The same value of $K_{i j}$ represents the fully symmetric junctions with $\Psi / \chi$ and $(\Psi / \chi)^{-1}$.

Several possibilities in order to take into account this symmetry in the statistical analysis are shown in Table 5. Two options have been considered. On the one hand the symmetrisation of the data. It means that a value of $K_{i j}$ obtained by a single computation is assigned to two different junctions with $\Psi / \chi$ and $(\Psi / \chi)^{-1}$. By doing so, the data 
is exactly symmetrical. It is indicated in Table 5 as 'symmetric data'. On the other hand the regression formula can be symmetrised by imposing $C_{1}=C_{3}=0$. This is indicated in Table 5 as 'symmetric formula'.

By considering cross combinations of these two options of imposing (or not) the symmetry, four different regression situations are analysed. In the first one, nothing is done (this is the one used in the plots). The regression coefficients $C_{1}$ and $C_{3}$ take a value in the statistical analysis. But it can be seen that it is in general much smaller than $C_{2}$ (the proximity to 0 is a kind of control on how symmetric the computed data is). Moreover the effects of $C_{1}$ and $C_{3}$ most probably compensate each other. A possible explanation of the light asymmetry of the computed data is the force excitation positions (which are not perfectly symmetric), the different combination of dimensions and the post-processing procedure. The increase of junctions included in the analysis would probably improve this aspect.

The other three situations (non-symmetric data with symmetric formula, symmetric data with symmetric formula and symmetric data with non-symmetric formula) provide exactly the same result (note that $C_{1}$ and $C_{3}$ are exactly null in the case of symmetric data with non-symmetric formula). Moreover, the difference of these three options with the first situation (non-symmetric data with non-symmetric formula) in terms of $R^{2}$ and Standard error is almost null. It means that the symmetry of the computed data is already quite acceptable and that the statistical analysis without imposing the symmetry, already leads to a quite symmetrical result.

\subsection{Thin and thick plate theo- ries}

As mentioned in Section 2, the spectral shell elements considered are formulated in terms of the thin (Kirchhoff) plate theory. This is the most standard and extended theory in order to simulate the bending behaviour of plates. For this reason it was considered here: to use the most common among all possible options.

However, the materials and junction dimensions used in Section 3 are representative of heavy junctions. And it is needed to check, at least for the thickest junctions, which are the differences if thick (Mindlin) plate theory is considered. According to the criteria proposed in [15], the limit frequency in the use of thin shell theory (plane stress) for quasi-longitudinal waves is above the frequency range considered in this study for all the materials in Table 2 and thicknesses. Consequently, the quasi-longitudinal waves are properly described by the spectral elements used here. This is not the case for the limit frequency of bending waves for which differences around $10 \%$ in terms of wave velocity can already be found at $500 \mathrm{~Hz}$ for the thickest junctions considered $(0.3 \mathrm{~m})$. The most critical material for this aspect is the aerated concrete but not very large differences between all the other materials exist.

A comparison between the use of thin and thick shell theories for some of the junctions is done by means of the FEM software CodeAster [19]. Table 11 shows the results of four different T-junctions made of 'Aerated concrete 2' material with constant thickness in all the parts. Similar results are found for the other materials. The output is the spatial averaged displacement modulus (in the direction orthogonal to the plate). The curve has been softened by making the average of five contiguous frequencies. This is a compromise between a third-octave frequency band average that would mask the differences (i.e. the small change in the position of some eigenfrequencies due to the differences in wavenumber between both theories would not noted because they are in the same band) and a frequency response curve without any average and with oscillations due to the modal 
response that would also make difficult to see some difference and obtain clear conclusions.

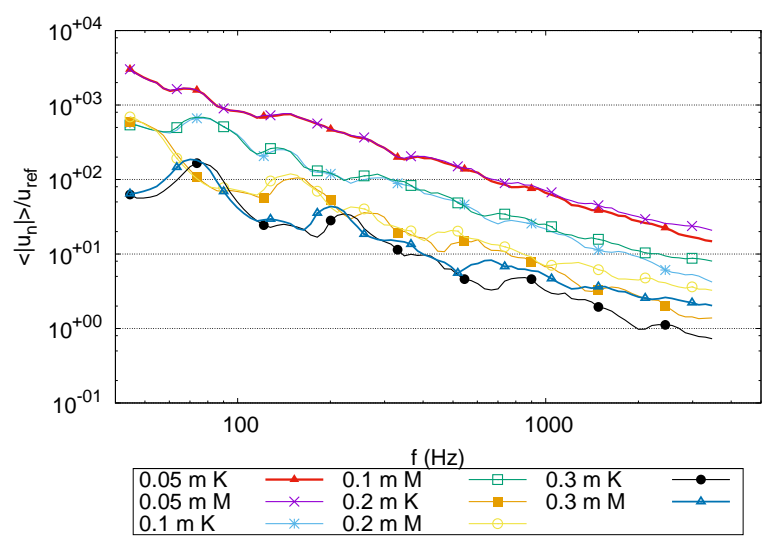

(a)

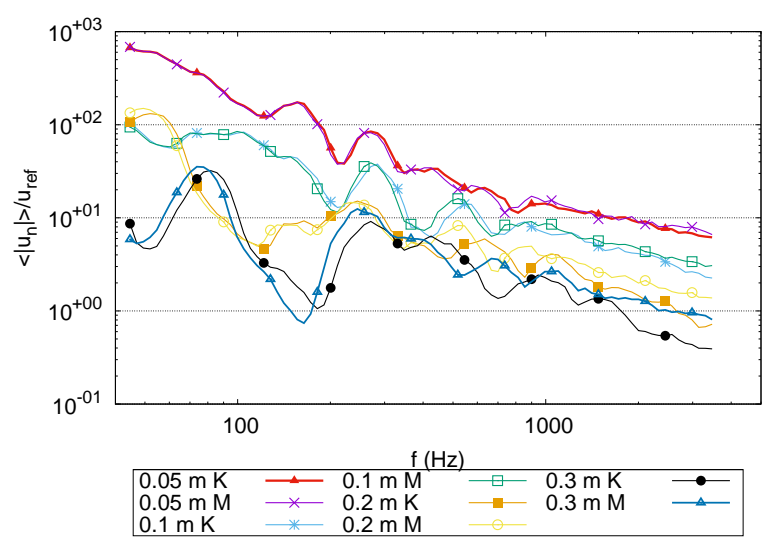

(b)

Figure 11: Averaged displacement field in the two aligned branches of a T-junction: (a) zone 1 ; (b) zone 3 . A unit point force acting on zone 1 is considered. The reference displacement $u_{\text {ref }}$ is taken as $10^{-10} \mathrm{~m}$.

Some differences can be seen above 1000 $\mathrm{Hz}$ and for the thickest junctions $(t=0.2$ and $0.3)$. However, they are not large enough in order to affect the averaged outputs that are the goal of this research. Moreover, the differences appear also in the same sense (larger displacement for the thick shell theory in all the zones of the junction). It is less influencing because $K_{i j}$ requires the vibration level difference. This is a ratio of velocities which tends to cancel this difference between both theories.
For all these, it can be concluded that the use of thin plate theory is perfectly valid in this research with the chosen materials and set of thicknesses.

\subsection{Applicability of the research to real junctions}

The model used in this paper deals with ideal junctions that are homogeneous and with isotropic material properties. Moreover, it is assumed that the junctions can be considered as isolated. The applicability of the results shown here to real junctions is most probably acceptable as far as the junction typology is close to the hypotheses of the model. Furthermore, small variations in plate dimensions or imperfection would most probably increase the $K_{i j}$ values presented in this paper; however, lower $K_{i j}$ values allows to be on the safe side when predicting the acoustic performance of the building. A probabilistic approach could actually be applied in order to take into account the uncertainty aspects associated to the material properties, dimensions and assembly conditions following methodology introduced by Soize [32]. A range of variability of $K_{i j}$ predictions can then be established.

\section{Conclusions}

The vibration reduction index of some 'less studied' junction types has been computed. As done in previous works [13], the used strategy tries to be general enough in order to provide design guidelines. Some combinations of typical materials and junction dimensions commonly found in buildings that have heavy junctions are considered.

When comparing with the available formulations and results for standard junctions, meaningful differences are found. This makes it evident that the studied junctions require a specific formulation that better describes 
their behaviour.

Apart from the regression formulas, the main findings of the research are:

1. The paths $2-4,2-5$ and $3-4$ of the $\mathrm{H}-$ junction are very specific of that junction type. It is difficult to explain them based on the $\mathrm{X}$-junction, whose formulas are approximately valid to provide some lower bound of $K_{i j}$. The spread of the results due to the leaves separation $d$ is important and makes it difficult to find a precise representation of the numerical data with a simple formula.

2. The paths $2-3$ and $3-1$ in the $\mathrm{H}-$ junction can be properly explained by means of the equivalent $\mathrm{X}$-junction. The results are very similar and the influence of the leaves separation $d$ could be neglected.

3. In the junctions which have non-orthogonal parts, the higher values of $K_{i j}$ are always found for the right-angle case. $K_{i j}$ can decrease approximately $1 \mathrm{~dB}$ per each $10^{\circ}$ of angle variation.

4. The only situation in which the angle formed by the junction parts was not influencing the vibration reduction index is the straight transmission $K_{13}$ at high frequencies.

5. In the asymmetrical junctions that have been considered, meaningful (i.e. $\geq 2 d B$ ) differences with the standard symmetrical junctions where only found for large $(>3)$ or $\operatorname{small}(<0.6)$ values of $\Psi / \chi$.

6. The output $K_{i j}$ has a large variability not only due to the different sets of data (material parameters, frequency, dimensions) considered but also due to the modal response of the junctions at some frequencies, the excitation type and the procedure to define and compute $K_{i j}$ itself (it is a quite post-processed output, i.e. not simply a point displacement). This leads sometimes the statistical approximation of the results to have a large standard error. For those cases when the standard error is larger than three (which are only some junctions and at low frequencies) the proposed formulas can lead to large differences when compared to an single junction. It could maybe be interesting to simulate the specific junction. For all the other situations, the proposed formulas provide a good estimation of $K_{i j}$ averaged trend with almost null effort.

\section{Acknowledgements}

Authors acknowledge financial support from the Research and Development Department of CSTB Carnot Institute. Free software has been used (Ubuntu environment, gcc compiler, gnuplot, xfig, Kyle, Qt-creator, Gmsh [33], Python, etc.). LaCàN research group is grateful for the sponsorship/funding received from Generalitat de Catalunya (Grant number 2014-SGR-1471).

\section{A Tables with the regres- sion formulas coefficients}

This appendix summarises the statistical analysis performed in order to fit a simple multilinear equation to the results obtained with SFEM. Each table corresponds to a different junction and includes coefficients for each of the three frequency ranges and the several paths considered. Tables 9, 10 and 11 are based on existent data while the other tables are the core of the present research. Eq. (6) is required in order to understand the meaning of the coefficients. $C_{d}$ and $C_{\theta}$ are omitted when not needed. 


\begin{tabular}{|c|c|c|c|c|c|c|c|c|}
\hline Path & Frequency & $C_{0}$ & $C_{1}$ & $C_{2}$ & $C_{3}$ & $C_{d}$ & $R^{2}$ & Std. Err. (dB) \\
\hline \multirow{4}{*}{$2-5$} & Low & 15.57 & 18.62 & 6.63 & 0.75 & 0.23 & 0.93 & 3.8 \\
\hline & Mid & 9.65 & 12.75 & 4.36 & 0.77 & 0.07 & 0.91 & 3.0 \\
\hline & High & 5.79 & 8.66 & 1.67 & -0.07 & 0.02 & 0.94 & 1.6 \\
\hline & Low & $\overline{8} . \overline{7} \overline{4}$ & $-\overline{1} . \overline{1} \overline{8}$ & $\overline{4} . \overline{7} \overline{7}$ & $\overline{0.73}-$ & $-\overline{0} . \overline{1}$ & $\overline{0.9} \overline{2}$ & $\overline{1} . \overline{2}$ \\
\hline \multirow[t]{3}{*}{$2-3$} & Mid & 8.4 & -0.99 & 4.44 & 0.67 & 0 & 0.96 & 0.7 \\
\hline & High & 7.34 & -1.02 & 4.38 & 0.7 & -0.01 & 0.98 & 0.6 \\
\hline & Low & $1 \overline{7} .6 \overline{3}$ & $\overline{9} . \overline{1}^{-}$ & $\overline{5} . \overline{3} \overline{9}$ & $\overline{0}^{-}$ & $\overline{0} . \overline{2} \overline{3}$ & $0 . \overline{6}$ & $\overline{4} . \overline{5}$ \\
\hline \multirow[t]{3}{*}{$2-4$} & Mid & 15.18 & 6.42 & 6.07 & 0.64 & 0.08 & 0.85 & 2.2 \\
\hline & High & 12.99 & 4.63 & 5.59 & 0.64 & 0.02 & 0.94 & 1.1 \\
\hline & Low & $1 \overline{1} .0 \overline{2}$ & $7 . \overline{12}$ & $\overline{1} . \overline{8} \overline{6}$ & $0 . \overline{75}$ & $\overline{0.2}$ & $\overline{0} . \overline{8} \overline{5}$ & $\overline{3} . \overline{6}$ \\
\hline \multirow[t]{3}{*}{$3-4$} & Mid & 6.98 & 5.1 & 2.94 & 0.13 & 0.13 & 0.88 & 2.5 \\
\hline & High & 6.3 & 1.8 & 2.92 & -0.03 & 0.1 & 0.84 & 1.7 \\
\hline & Low & $\overline{8} . \overline{7} \overline{7}$ & $\overline{10} \overline{2} \overline{5}$ & $\overline{5} . \overline{2} \overline{6}$ & $-0 . \overline{6} 8$ & $-\overline{0}$ & $\overline{0.9 \overline{8}}$ & $\overline{1} . \overline{5}$ \\
\hline \multirow[t]{2}{*}{$3-1$} & Mid & 8.69 & 9.28 & 2.88 & -0.47 & -0.01 & 0.97 & 1.6 \\
\hline & High & 4.52 & 6.16 & 1.46 & 0.06 & -0.05 & 0.88 & 2.2 \\
\hline
\end{tabular}

Table 4: Regression coefficients for the H-junction. 


\begin{tabular}{|c|c|c|c|c|c|c|c|c|}
\hline & Frequency & $C_{0}$ & $C_{1}$ & $C_{2}$ & $C_{3}$ & $C_{\theta}$ & $R^{2}$ & Std. Err. (dB) \\
\hline$\theta \leq 90^{\circ}$ & Low & -1.43 & 0.68 & 3.91 & -0.8 & 0.08 & 0.58 & 3.1 \\
\hline Non-symmetric data & Mid & -6.59 & 0.15 & 2.23 & -0.11 & 0.15 & 0.93 & 1.2 \\
\hline Non-symmetric formula & High & -7.12 & -0.14 & 2.13 & -0.05 & 0.13 & 0.94 & 1.0 \\
\hline $\bar{\theta} \leq \overline{9} 0^{\circ}-\cdots-\cdots-\cdots$ & Low & $-\overline{0} \overline{9} \overline{4}$ & --- & $\overline{2} . \overline{8} \overline{0}$ & $-\underline{--1}$ & $0 . \overline{0} 8$ & $\overline{0} \overline{.5} \overline{6}$ & $\overline{3} . \overline{1}$ \\
\hline Non-symmetric data & Mid & -6.52 & - & 2.10 & - & 0.15 & 0.93 & 1.2 \\
\hline Symmetric formula & High & -7.08 & - & 1.98 & - & 0.13 & 0.94 & 1.0 \\
\hline $\bar{\theta} \leq \overline{9} 0^{\circ}-\cdots-\cdots$ & Low & $-\overline{0} \overline{9} \overline{4}$ & $\overline{0} . \overline{0}$ & 2.8 & 0.0 & $\overline{0.0} \overline{8}$ & $\overline{0} . \overline{5} \overline{6}$ & $\overline{3} . \overline{1}$ \\
\hline Symmetric data & Mid & -6.52 & 0.0 & 2.10 & 0.0 & 0.15 & 0.93 & 1.2 \\
\hline Non-symmetric formula & High & -7.08 & 0.0 & 1.98 & 0.0 & 0.13 & 0.94 & 1.0 \\
\hline $\bar{\theta} \leq \overline{9} 0^{\circ}$ & Low & $-\overline{0} . \overline{9} \overline{4}$ & & 2.8 & & $0 . \overline{0} 8$ & $\overline{0} . \overline{5} \overline{6}$ & $\overline{3} . \overline{1}$ \\
\hline Symmetric data & Mid & -6.52 & - & 2.10 & - & 0.15 & 0.93 & 1.2 \\
\hline Symmetric formula & High & -7.08 & - & 1.98 & - & 0.13 & 0.94 & 1.0 \\
\hline$\theta>90^{\circ}$ & Low & 13.63 & 0.94 & 4.78 & -0.76 & -0.1 & 0.90 & 1.5 \\
\hline Non-symmetric data & Mid & 14.88 & 0.68 & 3.55 & -0.54 & -0.1 & 0.93 & 1.0 \\
\hline Non-symmetric formula & High & 12.59 & 0.12 & 2.7 & -0.28 & -0.07 & 0.78 & 1.5 \\
\hline $\bar{\theta}>\overline{9} 0^{\circ}-\cdots-\cdots-\cdots-\cdots$ & Low & $\overline{13.98}$ & & $\overline{3} . \overline{9} \overline{5}$ & & $-\overline{-0.1}$ & $\overline{0} \overline{8} \overline{8}$ & $\overline{1} . \overline{6}$ \\
\hline Non-symmetric data & Mid & 15.12 & - & 2.96 & - & -0.1 & 0.92 & 1.1 \\
\hline Symmetric formula & High & 12.72 & - & 2.29 & - & -0.07 & 0.77 & 1.5 \\
\hline $\bar{\theta}>\overline{9} 0^{\circ}-\cdots-\cdots$ & Low & 13.98 & $\overline{0} . \overline{0}$ & $\overline{3} . \overline{9} \overline{5}$ & 0.0 & -0.1 & $\overline{0} . \overline{8} \overline{8}$ & $\overline{1} . \overline{6}$ \\
\hline Symmetric data & Mid & 15.12 & 0.0 & 2.96 & 0.0 & -0.1 & 0.92 & 1.1 \\
\hline Non-symmetric formula & High & 12.72 & 0.0 & 2.29 & 0.0 & -0.07 & 0.77 & 1.5 \\
\hline $\bar{\theta}>\overline{9} 0^{\circ}-\cdots-\cdots-\cdots$ & Low & $\overline{13.98}$ & - & $\overline{3} . \overline{9} \overline{5}$ & - & -0.1 & $\overline{0} . \overline{8} \overline{8}$ & $\overline{1} . \overline{6}$ \\
\hline Symmetric data & Mid & 15.12 & - & 2.96 & - & -0.1 & 0.92 & 1.1 \\
\hline Symmetric formula & High & 12.72 & - & 2.29 & - & -0.07 & 0.77 & 1.5 \\
\hline
\end{tabular}

Table 5: Regression coefficients for the L-junction with variable angle. Four different options of statistical post-process imposing or not the symmetry are explored. 'Symmetric data' means that data have been symmetrised and 'Symmetric formula' means that coefficients $C_{1}$ and $C_{3}$ are imposed to be null. 


\begin{tabular}{|c|c|c|c|c|c|c|c|c|c|}
\hline & Path & Frequency & $C_{0}$ & $C_{1}$ & $C_{2}$ & $C_{3}$ & $C_{\theta}$ & $R^{2}$ & Std. Err. (dB) \\
\hline \multirow{4}{*}{$\theta \leq 90^{\circ}$} & & Low & 0.48 & -1.32 & 4.32 & -0.58 & 0.08 & 0.77 & 3.0 \\
\hline & $1-2$ & Mid & -5.1 & -1.48 & 3.16 & -0.33 & 0.15 & 0.92 & 1.4 \\
\hline & & High & -5.67 & -2.03 & 2.87 & -0.14 & 0.14 & 0.94 & 1.2 \\
\hline & & Low & $\overline{0} . \overline{8} \overline{2}$ & $\overline{6} . \overline{4} 2^{-}$ & $\overline{2} . \overline{8} \overline{7}$ & $-\overline{0.07}$ & $\overline{0} . \overline{0} \overline{9}$ & $\overline{0} . \overline{8} \overline{8}$ & $\overline{2} . \overline{8}$ \\
\hline \multirow[t]{3}{*}{$\theta \leq 90^{\circ}$} & $1-3$ & Mid & -3.08 & 6.33 & 2.12 & 0.02 & 0.08 & 0.93 & 1.9 \\
\hline & & High & -1.54 & 4.34 & 1.77 & 0.16 & 0.01 & 0.94 & 1.3 \\
\hline & & Low & $\overline{1} \overline{5} . \overline{7}$ & $-0.7^{-}$ & $\overline{4} . \overline{7} \overline{5}$ & -0.56 & $-\overline{0} . \overline{1} \overline{1}$ & $\overline{0.8 \overline{9}}$ & $\overline{1} . \overline{6}$ \\
\hline \multirow[t]{3}{*}{$\theta>90^{\circ}$} & $1-2$ & Mid & 17.25 & 0.1 & 4.16 & -0.61 & -0.12 & 0.91 & 1.4 \\
\hline & & High & 15.87 & 0.38 & 3.7 & -0.65 & -0.1 & 0.92 & 1.1 \\
\hline & & Low & $\overline{17} . \overline{44}$ & $\overline{6} . \overline{4} 2^{-}$ & $\overline{2} . \overline{8} \overline{7}$ & -0.07 & $-\overline{0} . \overline{0}$ & $\overline{0.8 \overline{8}}$ & $\overline{2} . \overline{8}$ \\
\hline \multirow[t]{2}{*}{$\theta>90^{\circ}$} & $1-3$ & Mid & 11.47 & 6.33 & 2.12 & 0.02 & -0.08 & 0.93 & 1.9 \\
\hline & & High & 0.96 & 4.34 & 1.77 & 0.16 & -0.01 & 0.94 & 1.9 \\
\hline
\end{tabular}

Table 6: Regression coefficients for the T-junction with variable angle.

\begin{tabular}{|c|c|c|c|c|c|c|c|}
\hline Path & Frequency & $C_{0}$ & $C_{1}$ & $C_{2}$ & $C_{3}$ & $R^{2}$ & Std. Err. (dB) \\
\hline \multirow{3}{*}{$1-3$} & Low & 7.21 & 2.49 & 4.96 & -0.92 & 0.98 & 0.7 \\
\hline & Mid & 3.63 & 2.75 & 2.03 & -0.19 & 0.73 & 2.1 \\
\hline & High & -0.22 & 1.99 & 1.95 & -0.19 & 0.85 & 1.2 \\
\hline & Low & $\overline{5} . \overline{4} \overline{7}$ & $\overline{2} . \overline{51}$ & 5.68 & $-\overline{1} . \overline{2} \overline{6}$ & $\overline{0.9 \overline{7}}$ & $\overline{0} . \overline{8}$ \\
\hline \multirow[t]{3}{*}{$1-2$} & Mid & 6.64 & 1.15 & 4.24 & -0.76 & 0.97 & 0.6 \\
\hline & High & 6.68 & 0.77 & 2.99 & -0.44 & 0.96 & 0.5 \\
\hline & Low & $\overline{5} . \overline{3} \overline{1}$ & $-6 . \overline{18}$ & $3 . \overline{97}$ & $-\overline{0} \overline{8} \overline{8}$ & $\overline{0.98}$ & $\overline{0} . \overline{8}$ \\
\hline \multirow[t]{2}{*}{$2-3$} & Mid & 6.50 & -3.49 & 2.79 & -0.68 & 0.95 & 0.7 \\
\hline & High & 6.58 & -2.90 & 2.13 & -0.65 & 0.96 & 0.5 \\
\hline
\end{tabular}

Table 7: Regression coefficients for the asymmetrical T-junction. 


\begin{tabular}{|c|c|c|c|c|c|c|c|c|}
\hline Path & Frequency & $C_{0}$ & $C_{1}$ & $C_{2}$ & $C_{3}$ & $R^{2}$ & Std. Err. (dB) & $\begin{array}{c}\text { Usual } \\
\text { Std. Err. (dB) } \\
\end{array}$ \\
\hline \multirow{4}{*}{$1-3$} & Low & 8.43 & 5.1 & 4.18 & 1.1 & 0.98 & 0.73 & 3.4 \\
\hline & Mid & 6.49 & 4.92 & 1.93 & 0.22 & 0.85 & 1.69 & 2.8 \\
\hline & High & 1.82 & 3.29 & 0.79 & 0.02 & 0.84 & 1.18 & 2.5 \\
\hline & Low & $\overline{7} . \overline{2} \overline{4}$ & $\overline{4} . \overline{4} \overline{1}$ & $\overline{5.35}$ & $-\overline{1} . \overline{2} \overline{5}$ & $\overline{0} . \overline{9} \overline{7}$ & $\overline{1} . \overline{1}$ & $\overline{2} . \overline{9}$ \\
\hline \multirow[t]{3}{*}{$1-4$} & Mid & 7.7 & 2.54 & 4.39 & -0.86 & 0.98 & 0.66 & 1.8 \\
\hline & High & 6.86 & 1.94 & 3.51 & -0.6 & 0.98 & 0.49 & 1.5 \\
\hline & Low & $\overline{7.7} 8^{-}$ & $-\overline{4} . \overline{34}$ & $-\overline{6.13}$ & $1 . \overline{4} 8$ & $\overline{0} . \overline{9} \overline{6}$ & $1 . \overline{35}$ & \\
\hline \multirow[t]{3}{*}{$2-4$} & Mid & 8.05 & -4.04 & 3.04 & 0.68 & 0.86 & 1.88 & - \\
\hline & High & 3.47 & -2.99 & 1.87 & 0.31 & 0.80 & 1.71 & - \\
\hline & Low & $7 . \overline{50}$ & $-\overline{5} . \overline{49}$ & $3 . \overline{92}$ & $-\overline{0} . \overline{9} \overline{8}$ & $\overline{0} . \overline{9} \overline{7}$ & $0 . \overline{8} 6$ & - \\
\hline \multirow[t]{2}{*}{$1-2$} & Mid & 7.94 & -3.85 & 2.62 & -0.59 & 0.97 & 0.55 & - \\
\hline & High & 6.66 & -2.55 & 2.45 & -0.67 & 0.95 & 0.51 & - \\
\hline
\end{tabular}

Table 8: Regression coefficients for the asymmetrical X-junction. The right column ('Usual Std. Err. $\left.(\mathrm{dB})^{\prime}\right)$ is the standard error if the regression formula for the symmetrical X-junction, with the coefficients in Table 10, is compared with the asymmetrical X-junction data.

\begin{tabular}{|c|c|c|c|c|c|c|c|}
\hline Path & Frequency & $C_{0}$ & $C_{1}$ & $C_{2}$ & $C_{3}$ & $R^{2}$ & Std. Err. (dB) \\
\hline \multirow{3}{*}{$1-3$} & Low & 6.97 & 6.47 & 4.28 & 0.02 & 0.99 & 1.0 \\
\hline & Mid & 3.51 & 7.22 & 2.25 & -0.03 & 0.94 & 1.8 \\
\hline & High & -0.28 & 4.22 & 1.65 & 0.19 & 0.91 & 1.6 \\
\hline & Low & $5 . \overline{3} 2$ & $-1 . \overline{77}$ & 5.84 & $-\overline{0} . \overline{2} \overline{5}$ & $\overline{0.96}$ & $\overline{1} . \overline{0}$ \\
\hline \multirow[t]{2}{*}{$1-2$} & Mid & 7.33 & -0.39 & 4.71 & -0.71 & 0.95 & 0.8 \\
\hline & High & 7.02 & -0.94 & 3.92 & -0.46 & 0.97 & 0.5 \\
\hline
\end{tabular}

Table 9: Regression coefficients for the standard T-junction.

\begin{tabular}{|c|c|c|c|c|c|c|c|}
\hline Path & Frequency & $C_{0}$ & $C_{1}$ & $C_{2}$ & $C_{3}$ & $R^{2}$ & Std. Err. (dB) \\
\hline \multirow{4}{*}{$1-3$} & Low & 8.29 & 9.23 & 5.17 & -0.57 & 0.99 & 0.8 \\
\hline & Mid & 6.2 & 8.33 & 2.82 & -0.13 & 0.96 & 1.7 \\
\hline & High & 2.23 & 5.94 & 1.86 & 0.17 & 0.92 & 1.9 \\
\hline & Low & $7 . \overline{58}$ & $0 . \overline{54}$ & $5 . \overline{7} 2$ & $-\overline{0} . \overline{8} \overline{6}$ & $\overline{0} . \overline{9} \overline{6}$ & $\overline{1} . \overline{0}$ \\
\hline \multirow[t]{2}{*}{$1-2$} & Mid & 8.77 & 0.35 & 4.4 & -0.59 & 0.96 & 0.8 \\
\hline & High & 7.38 & 0.59 & 4.49 & -0.63 & 0.98 & 0.6 \\
\hline
\end{tabular}

Table 10: Regression coefficients for the standard X-junction. 


\section{References}

[1] EN-12354. Building Acoustics: Estimation of the acoustic performance of buildings from the performance of elements. Technical Report 1-4, 1999-2000.

[2] E. Gerretsen. Calculation of the sound transmission between dwellings by partitions and flanking structures. Appl. Acoust., 12(6):413-433, 1979.

[3] E. Gerretsen. Calculation of airborne and impact sound insulation beteen dwellings. Appl. Acoust., 19(4):245-264, 1986.

[4] C. Hopkins. Measurement of the vibration reduction index, $\mathrm{K}_{i j}$ on freestanding masonry wall constructions. J. Building Acoustics, 6(3-4):235-257, 1999.

[5] C. Crispin, B. Ingelaere, M. Van Damme, and D. Wuyts. The vibration reduction index $\mathrm{K}_{i j}$ : Laboratory measurements for rigid junctions and for junctions with flexible interlayers. J. Building Acoustics, 13 (2):99-112, 2006.

[6] C. Crispin, M. Mertens, B. Blasco, B. Ingelaere, M. Van Damme, and D. Wuyts. The vibration reduction index $\mathrm{K}_{i j}$ :laboratory measurements versus predictions EN 12354-1 (2000). In The 33rd international congress and exposition on noise control engineering, Prague, 2004.

[7] S. Schoenwald, H.J. Martin, and E. Gerretsen. Aspects of the measurement of Kij at junctions of lightweight assembled structures. In CFA/DAGA'04, Strasbourg, 2004.

[8] C Hopkins. Determination of vibration reduction indices using wave theory for junctions in heavyweight buildings. Acta Acust. United Acust., 100(6):1056-1066, 2014.

[9] C. Hopkins. Sound insulation in buildings: linking theory and practice. In Acoustics 2012, Nantes, France, 2012.

[10] C. Hopkins and M. Robinson. On the evaluation of decay curves to determine structural reverberation times for building elements. Acta Acust. United Acust., 99(2):226-244, 2013.

[11] C. Hopkins and M. Robinson. Using transient and steady-state SEA to assess potential errors in the measurement of structure-borne sound power input from machinery on coupled reception plates. Appl. Acoust., 79:35-41, 2014.

[12] C. Crispin, L. De Geetere, and B. Ingelaere. Extensions of EN 12354 vibration reduction index expressions by means of FEM calculations. In InterNoise and Noise-con Congress and Conference Proceedings, volume 249, pages 5859-5868. Institute of Noise Control Engineering, 2014. 


\begin{tabular}{llcccccc}
\hline Path & Frequency & $C_{0}$ & $C_{1}$ & $C_{2}$ & $C_{3}$ & $R^{2}$ & Std. Err. $(\mathrm{dB})$ \\
\hline \multirow{3}{*}{$1-2$} & Low & 3.09 & 1.28 & 6.56 & -1.01 & 0.95 & 1.2 \\
& Mid & 5.57 & 0.88 & 4.09 & -0.65 & 0.97 & 0.6 \\
& High & 5.21 & 0.09 & 2.94 & -0.19 & 0.94 & 0.7 \\
\hline
\end{tabular}

Table 11: Regression coefficients for the standard L-junction.

[13] J. Poblet-Puig and C. Guigou-Carter. Using spectral finite elements for parametric analysis of the vibration reduction index of heavy junctions oriented to flanking transmissions and EN-12354 prediction method. Appl. Acoust., 99: 8-23, 2015.

[14] C. Hopkins, C. Crispin, J. PobletPuig, and C. Guigou-Carter. Regression curves for vibration transmission across junctions of heavyweight walls and floors based on finite element methods and wave theory. Appl. Acoust., 113: 7-21, 2016.

[15] C. Hopkins. Sound insulation. Routledge, 2012.

[16] J.F. Doyle. Wave propagation in structures: spectral analysis using fast discrete fourier transforms. Springer, New York, 1997. ISBN 0-387-94940-2.

[17] S. Gopalakrishnan, M. Ruzzene, and S. Hanagud. Spectral finite element method. In Computational Techniques for Structural Health Monitoring, pages 177-217. Springer London, 2011.

[18] S. Finnveden. Spectral finite element analysis of stationary vibrations in a beam-plate structure. Acta Acust. United Acust., 82(3):478-497, 1996.

[19] EDF. Code-Aster home page. http://www.code-aster.org, 2017.

[20] E. Reynders. Parametric uncertainty quantification of sound insulation val- ues. J. Acoust. Soc. Am., 135(4):19071918, 2014.

[21] J. Mahn and J. Pearse. On the uncertainty of the EN12354-1 estimate of the flanking sound reduction index due to the uncertainty of the input data. $J$. Building Acoustics, 16(3):199-231, 2009.

[22] International Organization for Standardization. ISO 10848-1:2006 acoustics - laboratory measurement of the flanking transmission of airborne and impact sound between adjoining rooms - part 1: Frame document. Technical report.

[23] J. Poblet-Puig. A modal-spectral model for flanking transmissions. J. Sound Vibr., 382:238-257, 2016.

[24] R.J.M. Craik. Damping of building structures. Appl. Acoust., 14(5):347359, 1981.

[25] B. Pfaff, J. Darrington, et al. GNU PSPP home page. http://www.gnu.org/software/pspp/, 2016 .

[26] A. Dijckmans. Vibration transmission across junctions of double walls using the wave approach and statistical energy analysis. Acta Acust. United Acust., 102 (3):488-502, 2016.

[27] A. Dijckmans. Wave based modeling of vibration transmission across junctions composed of rectangular single and double walls. Acta Acust. United Acust., 102 (6):1011-1026, 2016. 
[28] S. Schoenwald, B. Zeitler, and I. Sabourin. Analysis on structureborne sound transmission at junctions of solid wood double walls with continuous floors. In rth Forum Acusticum, 2014.

[29] F. Reis. Vibration transmission of asymmetric $\mathrm{t}$ form junctions. In Proceedings of 17th International Congress on Acoustics, Rome, 2001.

[30] F. Reis. Case studies in predicting airborne sound insulation in building. In Acústica, Congreso Iberoamericano de Acústica, $4^{\circ}$ Congreso Ibérico de Acústica, 35 Congreso Nacional de Acústica (Tecniacústica '04) y EAA Symposium, Guimaraes (Portugal), 2004.

[31] V. J Stauskis and M. Mickaitis. Vibration attenuation at asymmetric crossform joints of buildings. Journal of Civil Engineering and Management, 11 (2):129-135, 2005.

[32] C. Soize. Generalized probabilistic approach of uncertainties in computational dynamics using random matrices and polynomial chaos decompositions. Int. J. Numer. Meth. Engng., 81(8):939-970, 2010.

[33] C. Geuzaine and J.-F. Remacle. Gmsh: a three-dimensional finite element mesh generator with built-in pre- and postprocessing facilities. Int. J. Numer. Meth. Engng., 11(79):1309-1331, 2009. 